\title{
Modifications to the NASA SP-8072 Distributed Source Method II for Ares I Lift-off Environment Predictions
}

\author{
Jared Haynes \\ Qualis Corp. / ESTS Group \\ Huntsville, AL \\ Jeremy Kenny \\ NASA Marshall Space Flight Center \\ Huntsville, AL
}

\begin{abstract}
Ares I, also known as the Crew Launch Vehicle (CLV), is the crew launch component of Project Constellation, NASA's program to generate new vehicles for space flight and exploration. The first stage of Ares I will be powered by a five-segment Reusable Solid Rocket Motor (RSRMV), which is a modified version of the four-segment Reusable Solid Rocket Motor (RSRM) used for the Space Shuttle. During lift-off, the exhaust plume from the RSRMV will generate a considerable amount of sound, which will induce high vibration loads affecting the performance of the avionics instrumentation and/or the integrity of vehicle's structural components. In support of the Vehicle Integration group, the Fluid Dynamics Branch at Marshall Space Flight Center (MSFC) is responsible for predicting the acoustic environments for Ares I during lift-off. One of the tools useful for such a task comes from the NASA standard publication SP-8072 "Acoustic Loads Generated by the Propulsion System." Published in 1971, the SP-8072 monograph contains two Distributed Source Methods (DSM-1 and DSM-2) for predicting lift-off acoustics.
\end{abstract}

The DSM-1 method considers that the source of rocket noise in a given frequency band is limited to a confined region of the exhaust plume. By this assumption, a point source is assigned for each center frequency value of the third-octave band spectrum. The DSM-2 method is slightly more complex in that the exhaust plume is divided into finite regions or "slices." Using empirical curves provided in the SP-8072, each slice is assigned a total sound power as well as frequency content over a broad spectrum. The sound power generated by each slice is converted to a mean-square pressure level through the application of far-field acoustic theory, which implies that sound pressure varies inversely as the square of the distance from the source.

Generally, less than $1 \%$ of a rocket plume's kinetic energy is converted into acoustic energy as it interacts with the atmosphere and/or surrounding structures. Understanding that kinetic energy is directly proportional to mechanical stream power, the overall sound power generated by a rocket plume can be approximated as a percentage of the mechanical power. The overall sound power is distributed along the plume axis using empirical curves, where a spatial sound power density is non-dimensionalized using the aerodynamic reference length of the plume's potential core. The sound power density expression gives an analyst the freedom to choose the size and number of slices to represent the acoustic sources inside the plume. After the exhaust flow is divided into slices, the sound power generated by each slice 
is calculated from the sound power density. The frequency spectrum of each slice is obtained empirically from a spatially dependent spectral density of sound power, which is nondimensionalized by a "modified Strouhal number." The center of each slice is considered a spherically radiating monopole. The mean-square sound pressure level for each frequency band at any receiver position is calculated assuming that the sound pressure propagates spherically with additional empirical corrections. Realistically, the noise from rocket plumes does not propagate spherically; therefore, an additional empirical "directivity" term is necessary to adjust the shape of the sound pressure contour.

In this paper, three modifications are made to the DSM-2 reference data in an attempt to produce more accurate and physically realistic results. The first modification involves an adjustment to Eldred's relationship for approximating the potential core length in an exhaust plume. The distribution of a rocket's overall sound power along the exhaust axis is approximated using an aerodynamic reference dimension defined as the length of the plume's potential core region. In his monograph, Eldred provides an approximation for core length based on the subsonic jet studies of Lighthill and the supersonic jet studies of Anderson. Subsequent investigations of Eldred's work have shown two separate findings regarding core length definitions: 1) the proper aerodynamic reference length for this methodology is actually the "potential core" length, and 2) Eldred's core length approximation in the supersonic regime seems to be $\sim 2 \mathrm{x}$ too large when compared with current data. Using data from a 1967 study by Shirie, this paper shows that the discrepancy between Eldred's relation and the majority of recent measurements may be contributed to shock patterns inside the plume. The presence of oblique shocks, Mach disks, and expansion waves acts to increase the turbulence intensity in the exhaust flow, effectively shortening the length of the laminar "potential" core as well as the length of the "supersonic" core. Shirie's data implies that only in the rare instance that a shock-free, perfectly expanded flow can be achieved, the core lengths match those of Eldred. In all other conditions, along with some reported as perfect expansion, the core lengths are roughly half as long as Eldred's relationship predicts. The analysis in this paper utilizes the Varnier core length approximation for Ares I lift-off acoustic environments, which are generated by an under-expanded RSRMV.

A second modification is made to the way sound sources are distributed downstream of a plume deflection. In the DSM-1, Eldred provides source distribution curves for plumes experiencing no deflection, deflection through an open scoop, and deflection into a closed bucket. His data indicate that sources of sound are generated well downstream of the nozzle exit when the exhaust flow is unimpeded. However, when the exhaust flow is redirected by a deflector, the plume structure is affected such that sound sources appear much closer to the nozzle exit. Interestingly, in the DSM-2, Eldred does not acknowledge the affect of a deflector on axial sound source location; the consequence being that predictions using the DSM-1 and DSM-2 are highly inconsistent with each other, especially in a vertical launch configuration where plume deflection is present. The path of the plume and sound sources in the DSM-2 simply experience a change in direction, and there is no method of redistributing the sound power along the axial path of the plume downstream of a deflector. This implies that the plume structures, including the potential core, are preserved even after deflection takes place. The DSM-2 analysis in this paper utilizes a "terminated core" approach, in which the potential core is assumed to be destroyed upon impact with a deflector. The goal 
of this modification is to treat the plume deflection in a physically realistic manner and to adjust the sound source distribution accordingly.

A third modification made to the DSM-2 includes new directivity indices which are more suited to the first stage motor of the Ares I vehicle. Instead of using Eldred's empirical directivity data, which represent a curve-fit for motors of various sizes, a new set of directivity curves are calculated from acoustic data measured around an RSRM (Fig. 1). Although the RSRM and RSRMV have very similar exhaust flows, the non-dimensional Strouhal scaling is still utilized in this analysis for completeness.

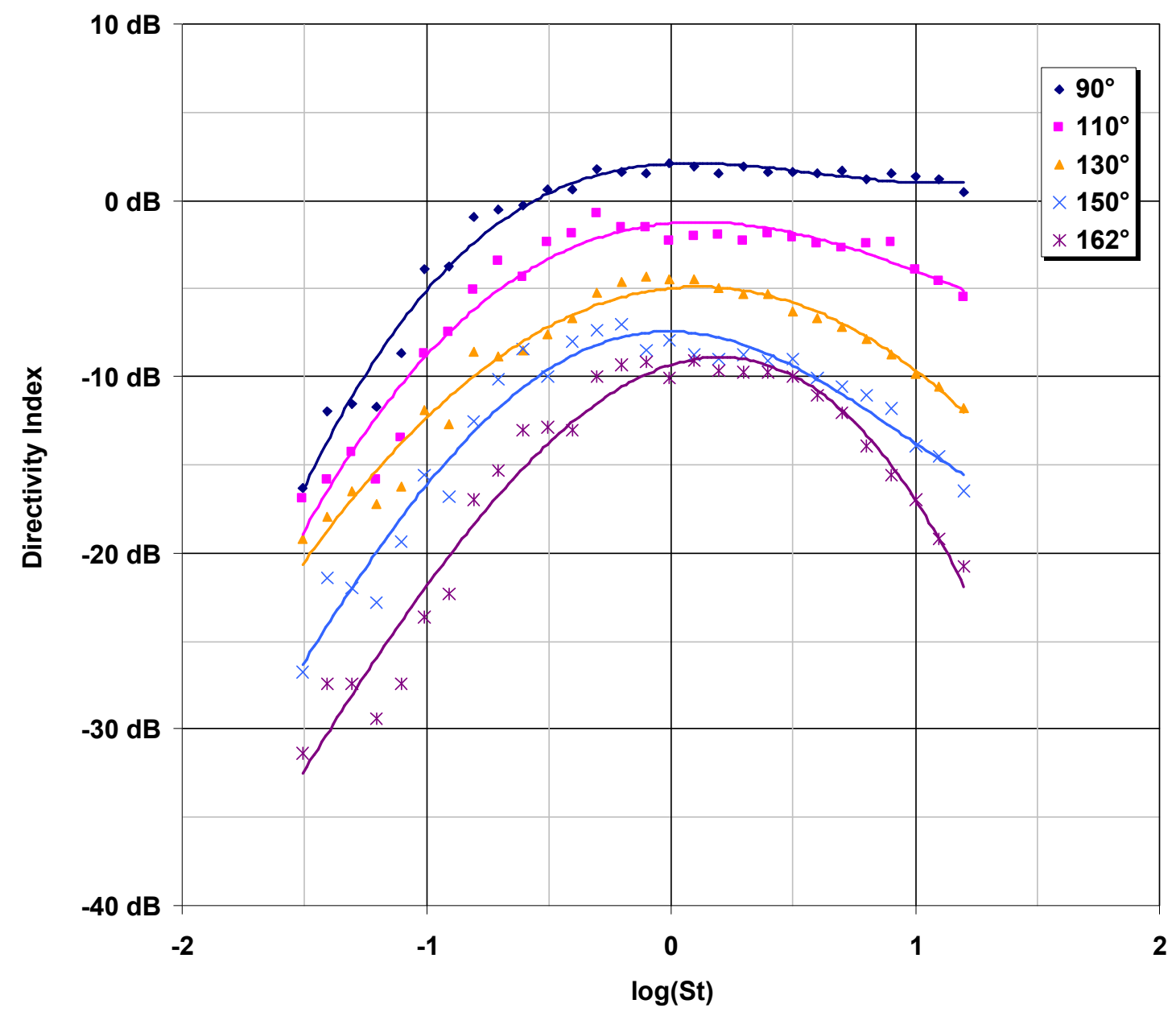

Figure 1 Directivity indices for a RSRM as a function of Strouhal number.

The effect of a shortened potential core that terminates upon deflection is an increase in sound pressure levels on the vehicle. Compared to Eldred's universal directivity data, the RSRM-specific directivity data flattens the sound pressure levels across the spectrum of frequencies. The peak at low frequencies appears lower to account for increased levels in the mid-to-high frequencies. Figure 2 shows predicted sound pressure levels in the $1 / 3$ octave 
band for three positions along the Ares I vehicle with distances measured from the nozzle exit.

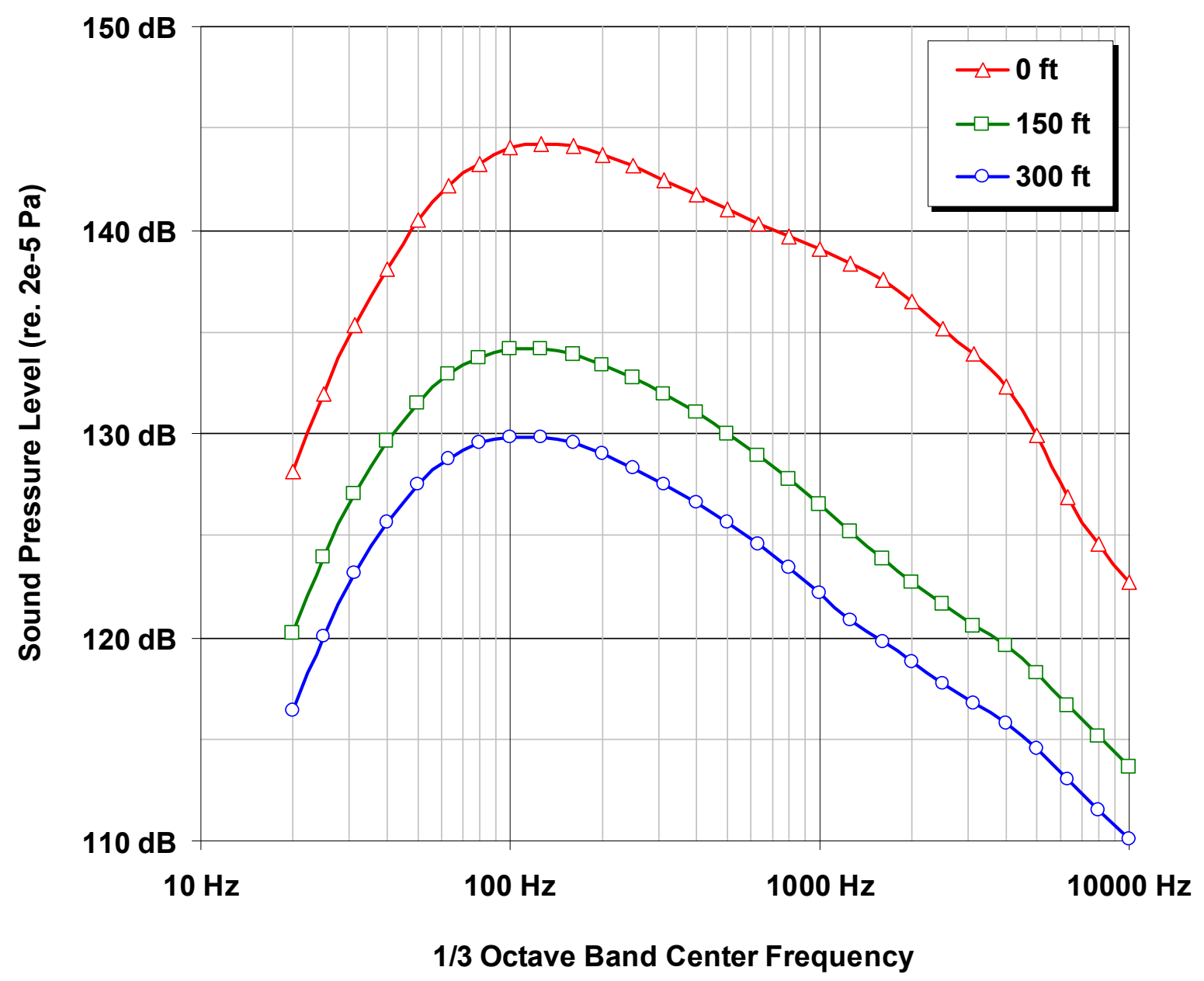

Figure 2 Sound pressure level predictions for Ares I with increasing distance from the nozzle exit.

In support of Ares I vehicle loads assessments, lift-off acoustic environment predictions were generated by the DSM-2 methodology. Three modifications were made to the methodology to better address plume core length, deflection, and acoustic directivity effects. Results show notable effects to the predicted sound pressure levels along the vehicle compared to unmodified predictions. 


\title{
Modifications to the NASA SP-8072 Distributed Source Method II for Ares I Lift-off Environment Predictions
}

\author{
Jared Haynes ${ }^{1}$ \\ Qualis Corporation / ESTS Group \\ NASA-Marshall Space Flight Center, Huntsville, AL, 35812 \\ R. Jeremy Kenny ${ }^{2}$ \\ NASA-Marshall Space Flight Center, Huntsville, AL, 35812
}

Lift-off acoustic environments for NASA's Ares I - Crew Launch Vehicle are predicted using the second source distribution methodology described in the NASA SP-8072. Three modifications made to the model include a shorter core length approximation, a core termination procedure upon plume deflection, and a new set of directivity indices measured from static test firings of the Reusable Solid Rocket Motor (RSRM). The modified sound pressure level predictions increased more than $5 \mathrm{~dB}$ overall, and the peak levels shifted two third-octave bands higher in frequency.

\section{Nomenclature}

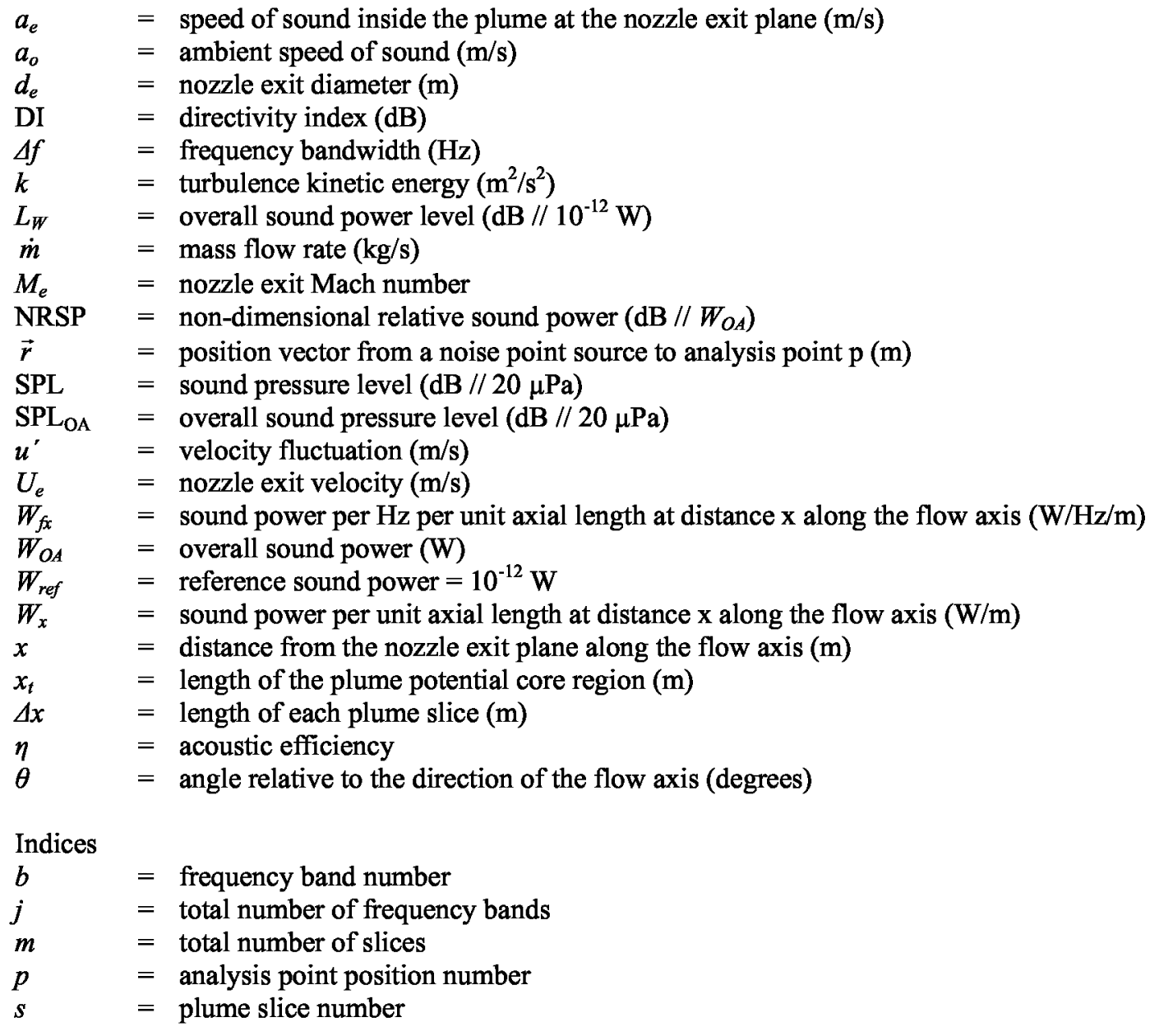

\footnotetext{
${ }^{1}$ Engineer, Qualis Corporation / ESTS Group, M/S ER42, NASA-MSFC, AL, 35812, Member, AIAA.

${ }^{2}$ Engineer, Acoustics and Stability Team, M/S ER42, NASA-MSFC, AL, 35812, Member, AIAA.
} 


\section{Introduction}

Ares I, also known as the Crew Launch Vehicle (CLV), is the crew launch component of Project Constellation, NASA's program to generate new vehicles for space flight and exploration. The first stage of Ares I will be powered by a five-segment Reusable Solid Rocket Motor (RSRMV), which is a modified version of the foursegment Reusable Solid Rocket Motor (RSRM) used for the Space Shuttle. During lift-off, the exhaust plume from the RSRMV will generate a considerable amount of sound, which will induce high vibration loads affecting the performance of the avionics instrumentation and/or the integrity of vehicle's structural components. In support of the Vehicle Integration group, the Fluid Dynamics Branch at Marshall Space Flight Center (MSFC) is responsible for predicting the acoustic environments for Ares I during lift-off. One of the tools useful for such a task comes from the NASA standard publication SP-8072 "Acoustic Loads Generated by the Propulsion System." Published in 1971, the SP-8072 monograph contains two Distributed Source Methods (DSM-1 and DSM-2) for predicting lift-off acoustics. ${ }^{1}$

\section{General Methodology}

DSM-1 and DSM-2 provide methods to predict the acoustic environments generated by rocket motors. The DSM-1 method considers that the source of rocket noise in a given frequency band is limited to a confined region of the exhaust plume. By this assumption, a point source is assigned for each center frequency value of the thirdoctave band spectrum. The DSM-2 method is slightly more complex in that the exhaust plume is divided into finite regions or "slices." Using empirical curves, each slice is assigned a total sound power as well as frequency content over a broad spectrum. The sound power generated by each slice is converted to a mean-square pressure level through the application of a far-field acoustic theoretical principle, which implies that sound pressure varies inversely with the square of the distance from the source.

The acoustic efficiency, $\eta$, for a rocket motor is an empirically derived quantity that represents the ratio of overall sound power to the kinetic power of the rocket exhaust. Generally, less than $1 \%$ of the kinetic power within a rocket plume is converted into sound power as it interacts with the atmosphere and/or surrounding structures. The overall sound power generated by a rocket plume is given in Eq. 1

$$
W_{O A}=\eta\left(\frac{1}{2} \dot{m} U_{e}^{2}\right)
$$

where $W_{O A}$ is the overall sound power, $\dot{m}$ is the mass flow rate, and $U_{e}$ is the nozzle exit velocity. Under most circumstances, acoustic power levels and pressure levels are represented in the decibel (dB) scale. The overall sound power is converted to the decibel scale by Eq. 2

$$
L_{W}=10 \log \frac{W_{o A}}{W_{r e f}}
$$

where $L_{W}$ is the overall sound power level, and $W_{\text {ref }}$ is the sound power reference value of $10^{-12} \mathrm{~W}$. The overall sound power is distributed along the plume axis using the empirical curve in Fig.12 in Eldred ${ }^{1}$, where a spatial sound power density, $W_{x}$, is non-dimensionalized using the aerodynamic reference length of the plume's potential core, $x_{t}$. The sound power density expression gives an analyst the freedom to choose the size and number of slices to represent the acoustic sources inside the plume. The total sound power produced by each slice, $s$, is calculated by Eq. 3

$$
L_{W, s}=L_{W}+10 \log \left(\frac{W_{x}}{W_{O A}} x_{t}\right)+10 \log \left(\frac{\Delta x}{x_{t}}\right)
$$

where $L_{W, s}$ is the sound power level of each slice and $\Delta x$ is the length of each slice. The frequency spectrum of each slice is obtained empirically from Fig. 13 in Eldred ${ }^{1}$. This figure represents a spatially dependent spectral density of sound power, which is non-dimensionalized by a "modified Strouhal number." The sound power, $L_{W, s, b}$, of each frequency band, $b$, in each slice is calculated by Eq. 4 


$$
L_{W, s, b}=L_{W, s}+10 \log \left(\frac{W_{f x}}{W_{x}} \frac{U_{e} a_{o}}{x a_{e}}\right)+10 \log \left(\frac{x a_{e}}{U_{e} a_{o}}\right)+10 \log \Delta f_{b}
$$

where $W_{f x}$ is the sound power per $\mathrm{Hz}$ per unit axial length along the flow axis, $a_{o}$ is the ambient speed of sound, $a_{e}$ is speed of sound inside the plume at the nozzle exit plane, $x$ is the distance from the nozzle exit plane along the flow axis, and $\Delta f_{b}$ is the frequency bandwidth of interest. The center of each slice is considered a spherically radiating monopole. The sound pressure level at any point of interest is calculated assuming that the sound power is dispersed along a spherical surface area. The radius of the sphere is simply calculated as the magnitude of the position vector, $\vec{r}$, from the source to the receiver. Realistically, the sound pressure levels from rocket plumes do not attenuate with spherical symmetry; therefore, an additional empirical "directivity" term is necessary to adjust the shape of the sound pressure contour. The conversion of sound power to sound pressure level for a given analysis point, $p$, is shown in Eq. 5

$$
\operatorname{SPL}_{s, b, p}=L_{r, s, b}-10 \log \left(4 \pi\left|\vec{r}_{s, p}\right|^{2}\right)+\operatorname{DI}_{b}\left(\theta_{s, p}\right)
$$

where $\operatorname{DI}_{b}\left(\theta_{s, p}\right)$ is the empirically measured directivity index for each frequency band, shown as a function of the angle with respect to the direction of exhaust flow. Figure 1 illustrates the problem geometrically.

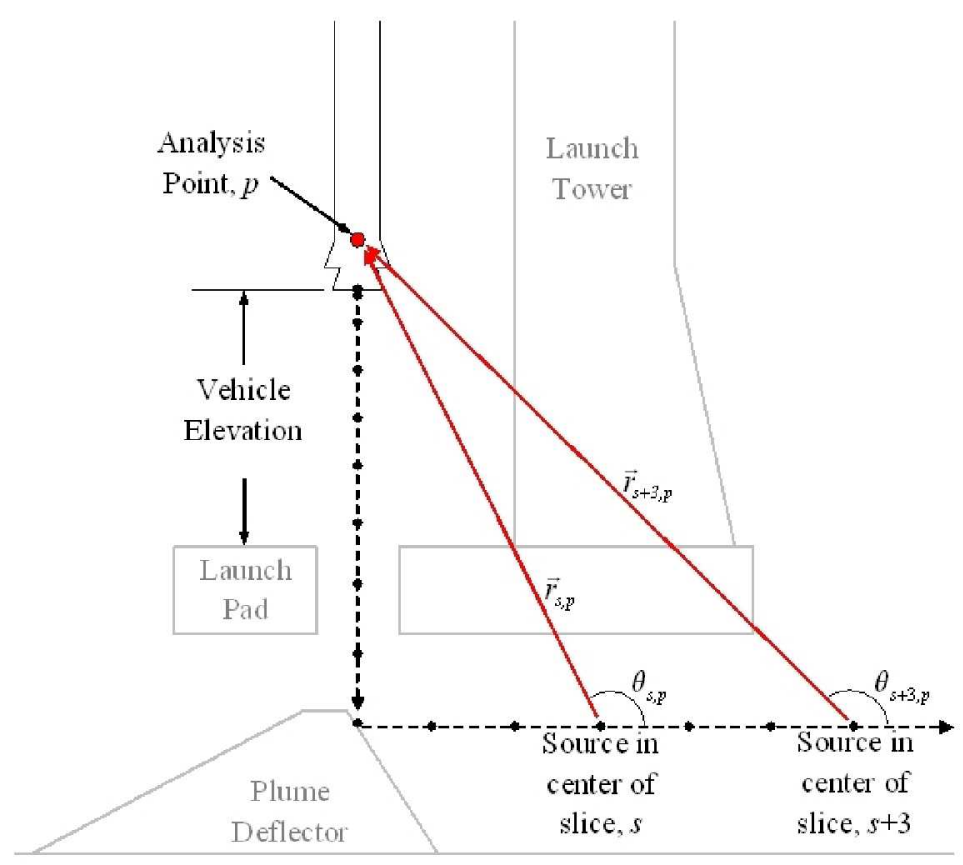

Figure 1. Illustration of DSM-2 analysis technique for sound attenuation and directivity.

The sound pressure level in each frequency band at each analysis point $\mathrm{SPL}_{b, p}$ is computed by logarithmic summation of contributions from a total $m$ number of slices in Eq. 6 .

$$
\mathrm{SPL}_{b, p}=10 \log \left(\sum_{s=1}^{m} 10^{\mathrm{SPL}_{s, b p} / 10}\right)
$$

The overall sound pressure level at any point, $p$, is computed by logarithmic summation of $\mathrm{SPL}_{b, p}$ contributions from the total $j$ number of frequency bands in Eq. 7. 


$$
\mathrm{SPL}_{\mathrm{OA}_{p}}=10 \log \left(\sum_{b=1}^{j} 10^{\mathrm{SP}_{b, p} / 10}\right)
$$

\section{Modifications}

\section{A. Potential Core}

The distribution of a rocket's overall sound power along the exhaust axis is approximated using the nondimensional relative sound power (NRSP) curve in Fig. 2. The power distribution along the axis of the plume is plotted with the aerodynamic reference dimension, $x_{t}$, defined as the length of the plume's potential core region. The calculation for NRSP is shown in Eq. 8.

$$
N R S P=10 \log \left(\frac{W_{x}}{W_{O A}} x_{t}\right)
$$

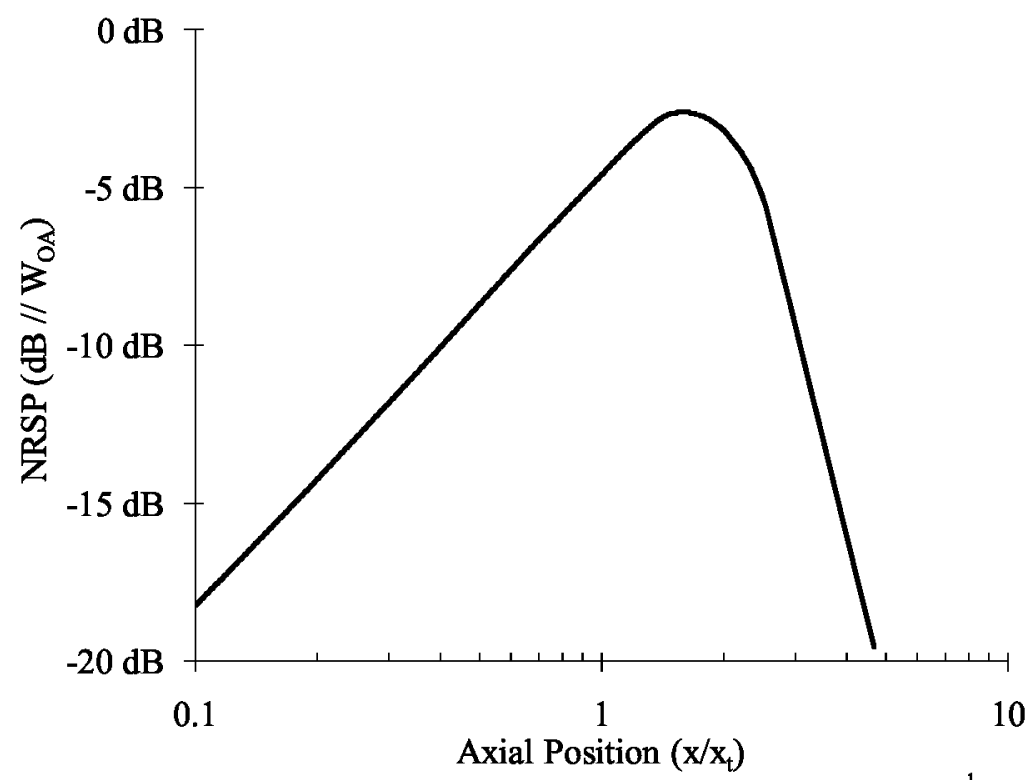

Figure 2. Source power distribution for standard chemical rockets. ${ }^{1}$

In his monograph, Eldred provides an approximation for core length based on the subsonic jet studies of Lighthill, 2,3 and the supersonic jet studies of Anderson. ${ }^{4}$ In the regime of supersonic flow, Eldred creates some confusion from use of the term "supersonic core" for the "potential core" region. To clarify this here forth, the "potential core" is defined as the core region of laminar flow, and the "supersonic length" represents the entire region of exhaust flow above Mach 1. Subsequent investigations of Eldred's work have shown two separate findings regarding some of the confusion: 1) the proper aerodynamic reference length for this methodology is actually the "potential core" length and 2) Eldred's core length approximation in the supersonic regime seems to be $\sim 2 \mathrm{x}$ too long when compared with current data ${ }^{5-7}$. Tam $^{8}$ notes that if the nozzle exit pressure deviates from the nozzle's designed flow condition, the exit conditions should be corrected for perfect expansion using isentropic, compressible flow relationships before approximating a core length. However, these calculations alone cannot correct for a factor of two in core length.

French research engineer, Jean Varnier, ${ }^{5,6}$ suggests that Eldred's relation (Eq. 9) shows disagreement with test data unless reduced by a factor of two (Eq. 10).

$$
\frac{x_{t}}{d_{e}}=3.45\left(1+0.38 M_{e}\right)^{2} \quad\{\text { Eldred }\}
$$




$$
\frac{x_{t}}{d_{e}}=1.75\left(1+0.38 M_{e}\right)^{2} \quad\{\text { Varnier }\}
$$

In these equations, $d_{e}$ represents the effective exit diameter of the exhaust flow, and $M_{e}$ is the Mach number of the exhaust flow at the nozzle exit.

A Reynolds Averaged Navier Stokes (RANS) Computational Fluid Dynamic (CFD) turbulence model can provide some insight into this problem. Turbulence is related to velocity fluctuations in a flow and can be calculated from the variable for turbulent kinetic energy, $k$. Figure 3 shows a contour of velocity fluctuations, $u$ ', for an underexpanded RSRM plume exhausting into an open space of standard atmospheric conditions. A close examination of this chart shows that the potential core, or region of very low turbulence, is roughly $24 \mathrm{~m}$ long. The axial flow remains supersonic for $57 \mathrm{~m}$ downstream of the nozzle exit. Using the CFD solution along with Fig. 2, the location of maximum sound power production should occur at $36 \mathrm{~m}$ along the flow axis. The y-axis in Fig. 3 represents a lineal sound power distribution calculated using the NRSP and the core length approximations of Varnier and Eldred. Interestingly, Eldred's max sound power is predicted to occur $75 \mathrm{~m}$ downstream of the nozzle, while Varnier's is located very close to $36 \mathrm{~m}$. This provides evidence that Eldred's core length equation may not be appropriate for non-ideally expanded flows. Thus, for Ares I lift-off acoustic environments, generated by an underexpanded RSRMV, it seems most reasonable to utilize Varnier's core length approximation.

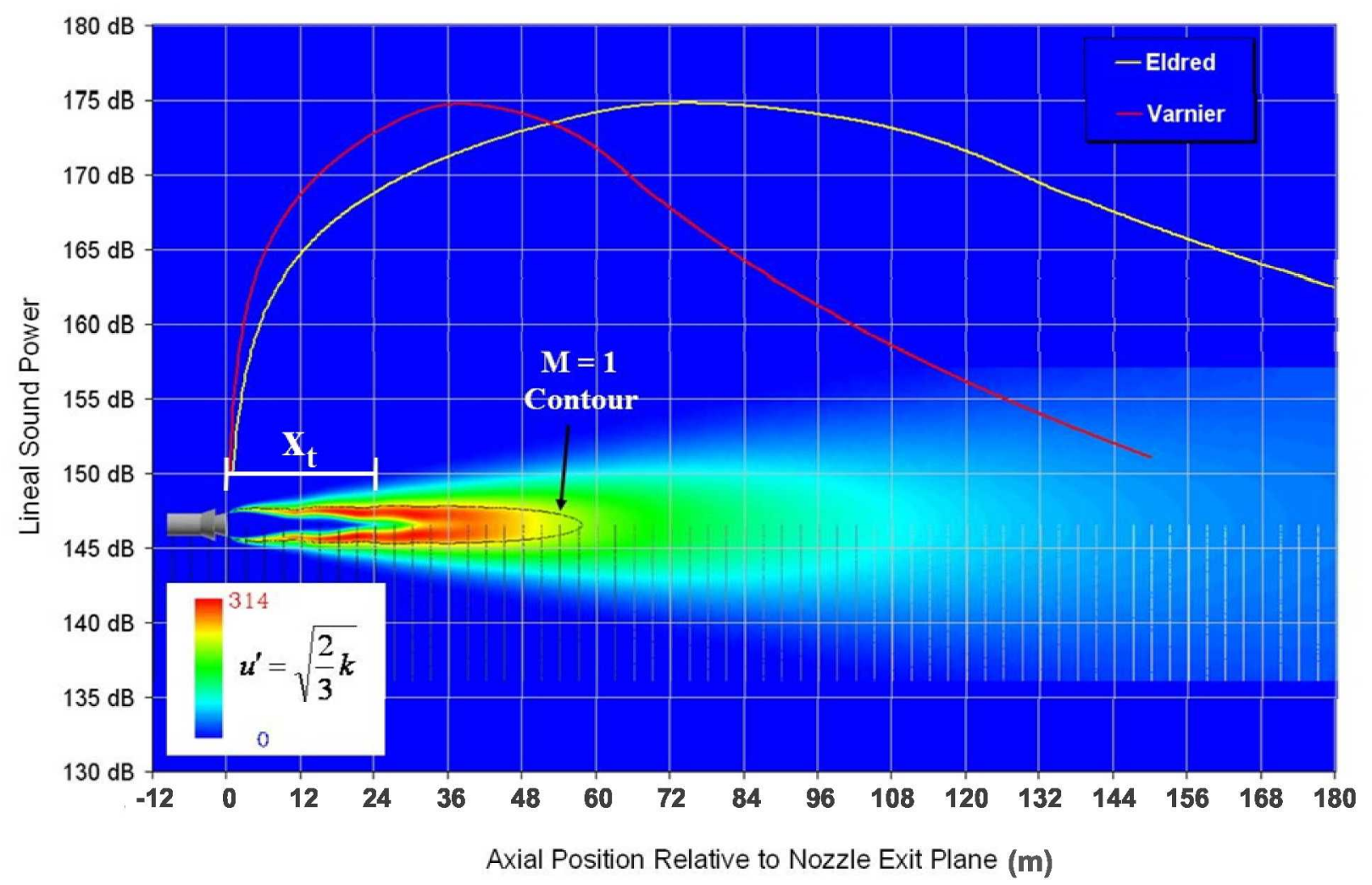

Figure 3. Comparison of Varnier and Eldred sound power distributions plotted along with turbulent velocity fluctuations from a RANS CFD solution for an RSRM plume. ${ }^{9}$

\section{B. Core Termination}

In the DSM-1, Eldred provides source distribution curves for plumes experiencing no deflection, deflection through an open scoop, and deflection into a closed bucket. The data in Fig. 4 indicate that sources of sound are generated well downstream of the nozzle exit when the exhaust flow is unimpeded. However, when the exhaust flow is redirected by a deflector, the plume structure is affected such that sound sources appear much closer to the nozzle exit. 


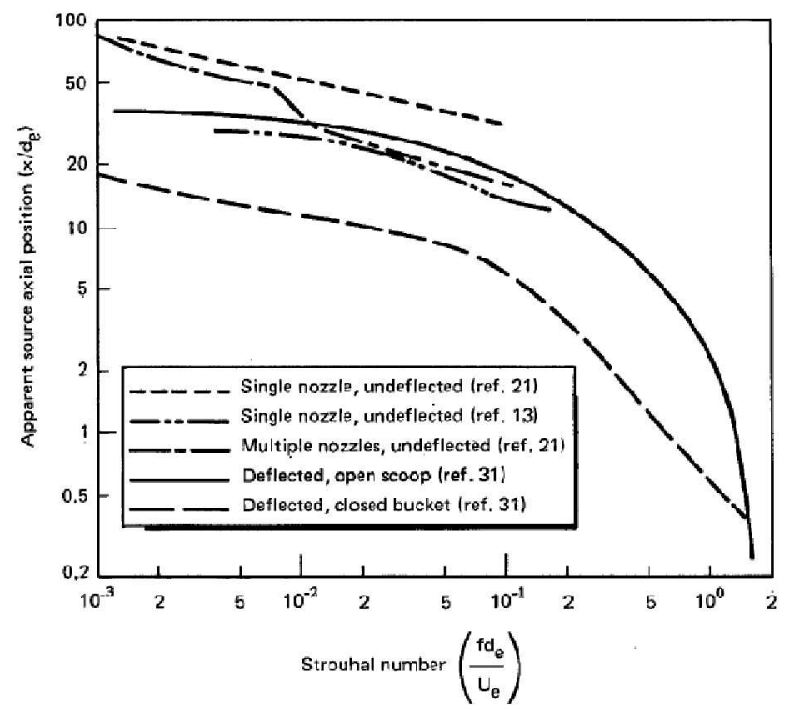

Figure 4. Axial location of apparent sources used in the DSM-1. ${ }^{1}$

Interestingly, in the DSM-2, Eldred does not acknowledge the effect of a deflector on axial sound source location; the consequence being that predictions from the DSM-1 are not consistent with those from the DSM-2, especially in a vertical launch configuration where plume deflection is present. The path of the plume and sound sources in the DSM-2 simply experience a change in direction, and there is no method of redistributing the sound power along the axial path of the plume downstream of a deflector. This implies that the plume structures, including the potential core, are preserved even after deflection takes place. Physically, it is already difficult to justify the presence of a laminar core inside a shock-containing plume, but assuming a sustained laminar core after an abrupt deflection seems even more unrealistic.

In order to examine the problem further, the same CFD turbulence model from section IIIA was used to observe qualitative characteristics for an RSRM plume impacting a deflector during launch. Similar to Fig. 3, a contour of velocity fluctuations was created in order to observe the laminar region of the exhaust flow. The CFD result, shown in Fig. 5, provides supportive evidence that the laminar region of the plume is greatly disturbed by the presence of a deflector. There is an abrupt increase in turbulence along the deflector surface, effectively terminating the region of exhaust flow that can be considered laminar. The approximate axial length of the laminar region, $x_{t}$, is indicated with a white arrow. According to Fig. 2, the apparent location for maximum sound power generation occurs at $1.5 \mathrm{x}_{\mathrm{t}}$, which appears to be very close to the bottom or slightly downstream of the deflection. After impacting the deflector, the flow has been turned $90^{\circ}$, and the increased turbulence levels are sustained inside the supersonic boundary. Extremely high turbulence occurs across the supersonic boundary, as well as the bottom of the deflector

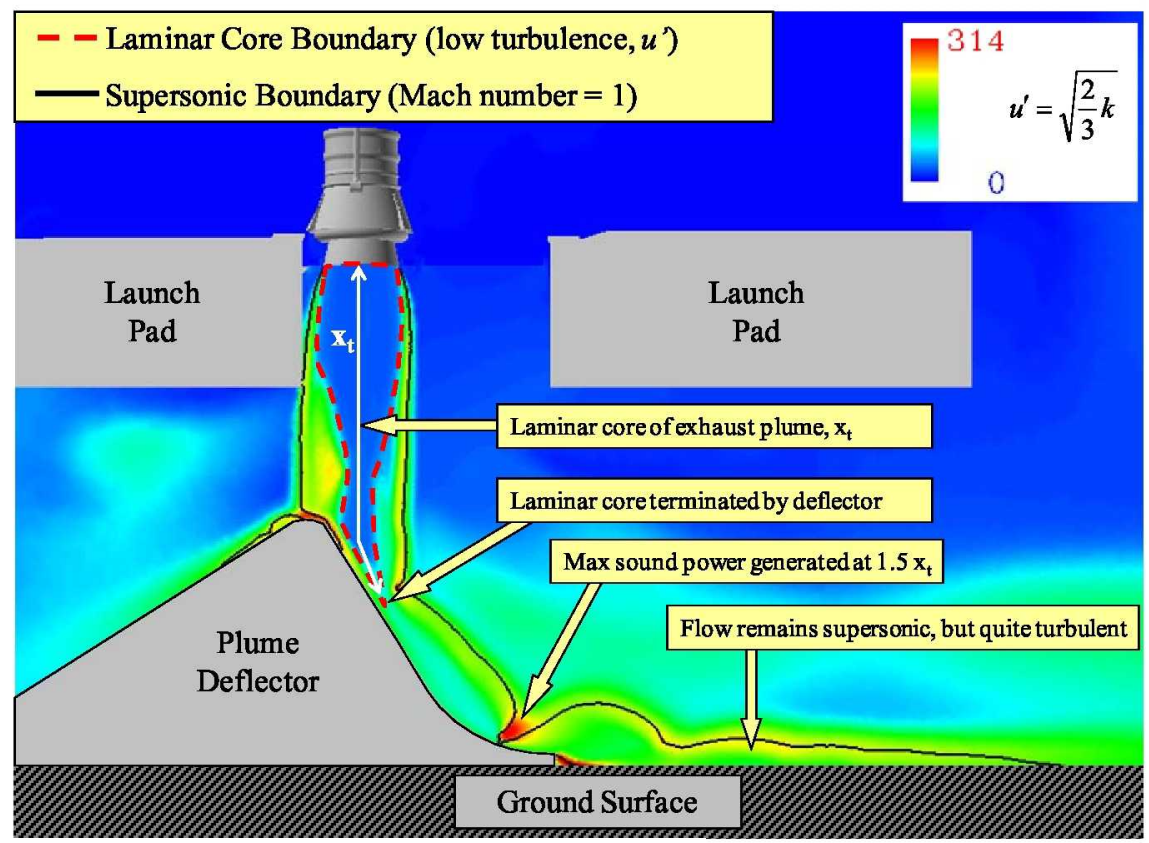

Figure 5. CFD solution of velocity fluctuations for a deflected RSRM plume. 
where shock patterns create strong discontinuities. The qualitative information gained from the CFD results can be used to modify the locations of sources in the DSM-2.

The DSM-2 analysis in this paper utilizes a "terminated core" approach, in which the potential core is assumed to be destroyed upon impact with a deflector (Fig. 6). The goal of this modification is to treat the plume deflection in a physically realistic manner and to adjust the sound source distribution accordingly using Fig. 2 .

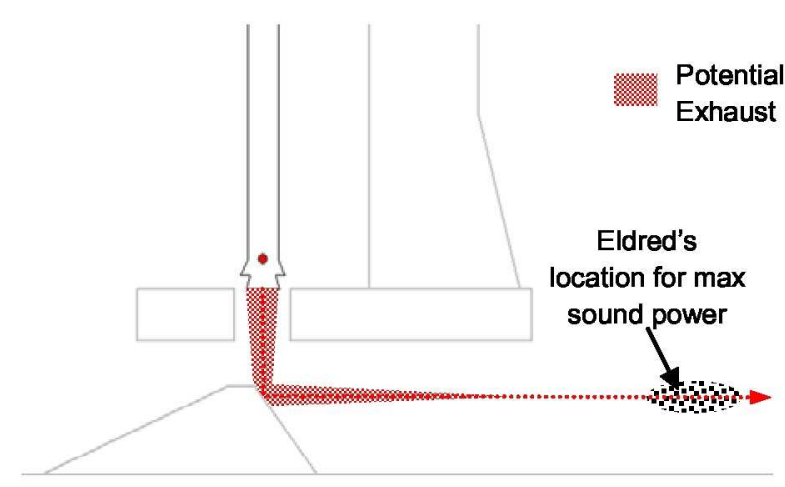

Eldred's Core Deflection
Potential Core of the

xhaust Plume

Figure 6. Illustration of sound power shift with a terminated core.

The new "terminated core" approach is validated using lift-off acoustic data on the Saturn V. In Figs. 7 and 8 , sound pressure levels from five Saturn V flights are plotted in third octave band spectra. The data is compared with two predictions from the DSM-2: 1) the original Eldred method where the potential core changes direction at deflection, but the length and structure are preserved; and 2) the terminated core approach, where the potential core length is severed upon impact with a deflector. The comparison shows that the shorter core length causes the sound pressure levels to increase significantly, as well as shift towards higher frequencies. With a shorter core, the sources of sound are located closer to the vehicle, reducing the attenuation distance from source to receiver. The frequency shift is caused by a redistribution of the power spectrum variable $W_{\mathrm{fx}}$ from Eq. 4 . Figures 7 and 8 show strong evidence that the core termination approach is an important improvement to Eldred's original methodology.

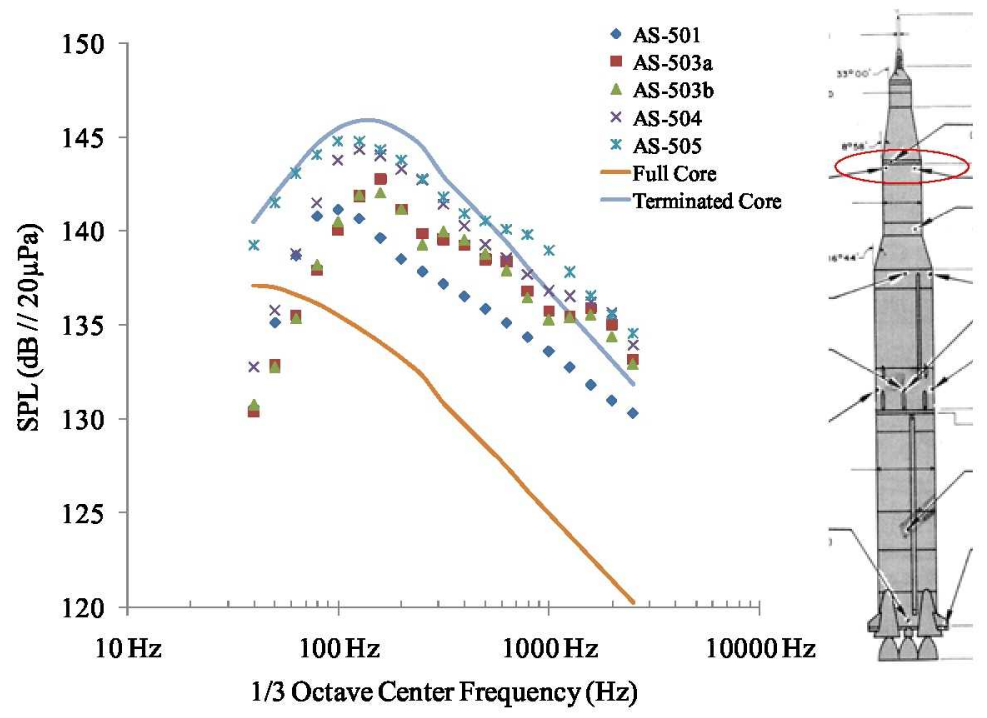

Figure 7. Saturn V DSM-2 comparison between terminated core and Eldred's full core approach, plotted with Saturn V lift-off acoustic data measured at $85 \mathrm{~m}$ from the nozzle exit. ${ }^{10,11}$ 


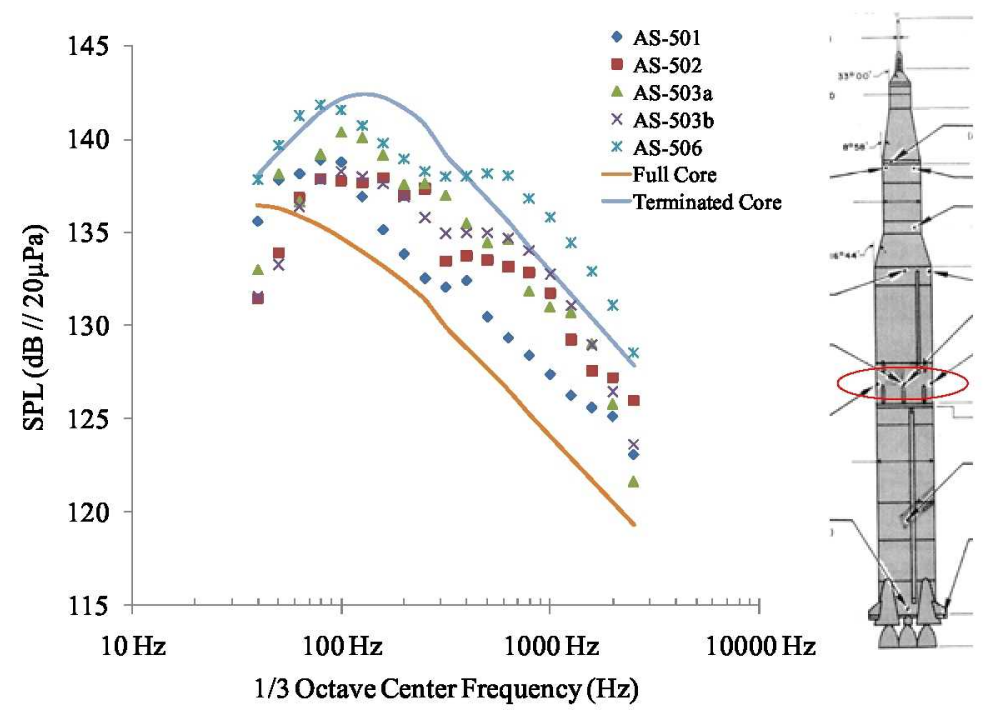

Figure 8. Saturn V DSM-2 comparison between terminated core and Eldred's full core approach, plotted with Saturn V lift-off acoustic data measured at $45 \mathrm{~m}$ from the nozzle exit. ${ }^{10,11}$

\section{Directivity}

A third modification made to the DSM-2 includes new directivity indices which are specifically suited to the first stage motor of the Ares I vehicle. Understanding the directivity of rocket noise is crucial for acoustics prediction models. Although the sound waves physically propagate in a relatively spherical manner, the sound pressure levels along the spherical spreading surface do not. For some frequencies, the position of a receiver relative to the direction of exhaust flow can make the difference of $15 \mathrm{~dB}$ from directivity alone. Instead of using Eldred's empirical directivity data, represented with curve-fits for motors of various sizes, a new set of directivity curves are calculated from acoustic data measured around a RSRM.

Recently, members of the MSFC Fluid Dynamics Branch and Wyle Labs measured farfield acoustic data during a series of three RSRM horizontal static tests conducted in Promontory, Utah. The test motors included the Technical Evaluation Motor 13 (TEM-13), Flight Verification Motor 2 (FVM-2), and the Flight Simulation Motor 15 (FSM-15); however, only the FVM-2 and FSM-15 tests were considered for this work because the TEM-13 nozzle duty cycle (nozzle gimbaling) was uncommon with the standard test procedure. The farfield measurements were intended to provide inputs for acoustic prediction models such as those described in the NASA SP-8072. For the purposes of the DSM-2, the measurements were used to calculate the acoustic efficiency and the directivity indices.

The farfield test configuration included microphones placed in a circular array centered about the RSRM nozzle exit. Data were collected at a $305 \mathrm{~m}$ radius from the RSRM nozzle, and included angular positions of $26^{\circ}, 36^{\circ}, 46^{\circ}, 56^{\circ}, 66^{\circ}, 76^{\circ}, 86^{\circ}, 96^{\circ}, 106^{\circ}$, $126^{\circ}$, and $146^{\circ}$ from the exhaust axis. During the time period that the data were processed, the nozzle and exhaust axis were vectored $4^{\circ}$ towards the microphones; therefore, each microphone angular position was reduced by $4^{\circ}$ in post processing calculations (Fig. 9). The measured sound pressure levels are plotted in Fig. 10 for a wide range of third-octave frequency bands. The directivity indices represent the difference between the sound pressure level at each microphone and the average sound pressure level across the array of $n$ microphones (Eq. 11), and are plotted in Fig. 11. These values were inserted into the DSM-2 model to represent the predicted directional characteristics of the Ares I exhaust plume noise.

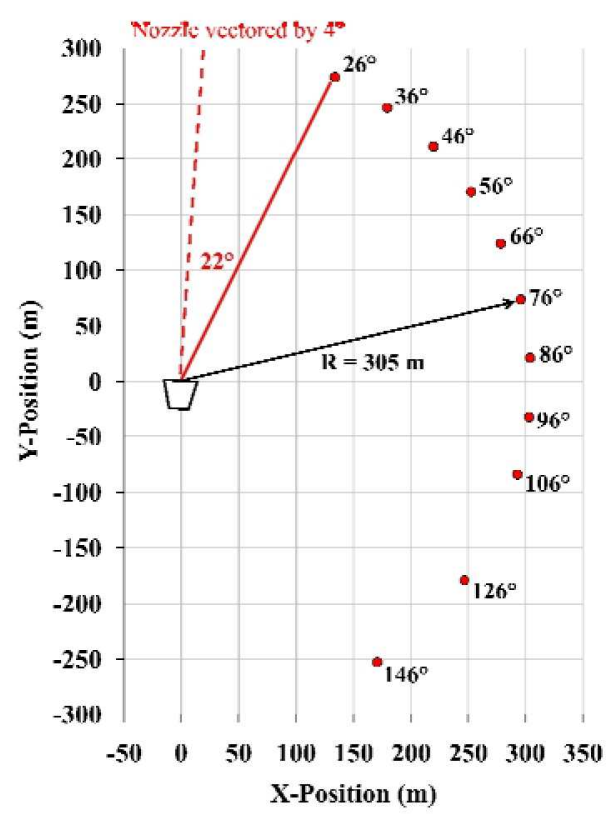

Figure 9. Microphone layout for RSRM acoustic measurements. 


$$
\mathrm{DI}_{b, \theta}=\mathrm{SPL}_{b, \theta}-10 \log \left(\frac{1}{n} \sum_{i=1}^{n} 10^{\mathrm{SP}_{b}, \theta_{i} / 10}\right)
$$

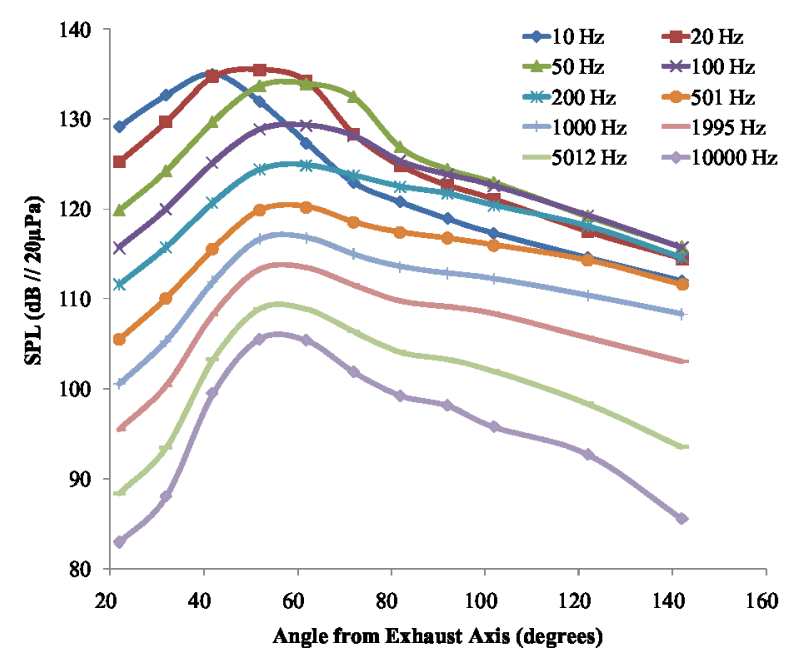

Figure 10. SPLs from RSRM farfield measurements.

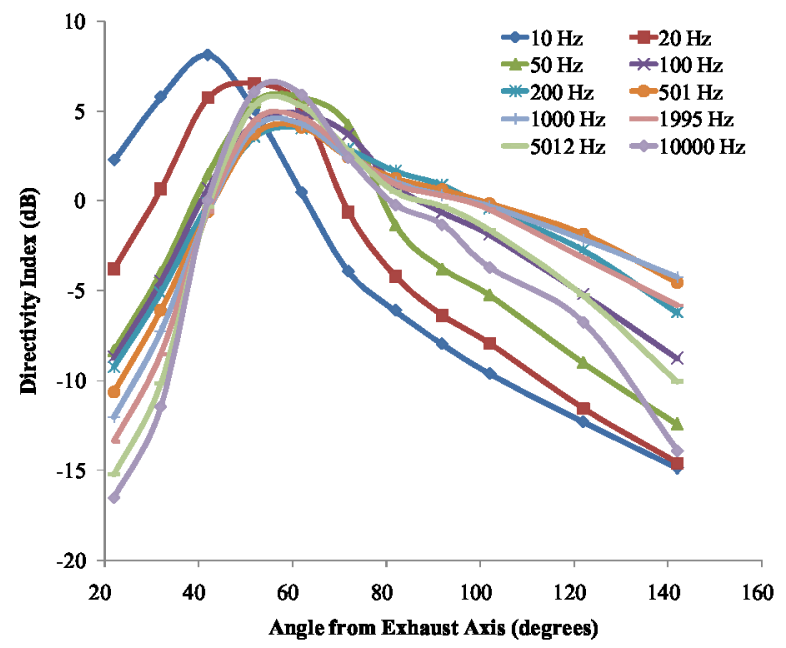

Figure 11. RSRM Directivity Index.

In comparison with Eldred's curves, the RSRM data show both similarities and contrasts in directivity trends. The lower frequencies contain the highest directional patterns, and the peaks shift to higher angles as frequency increases. These characteristics are consistent with those found in Eldred's data. The most significant contrast with Eldred's data exists for high frequency content at large angles. Typically, as frequency increases, the noise becomes less directional, and the slope of SPLs decreases. The RSRM data in Fig. 10 show that the SPLs become less directional up to $500 \mathrm{~Hz}$, but then actually become more and more directional from $500 \mathrm{~Hz}$ to 10,000 Hz. This unexpected trend warrants further investigation in to possible terrain effects at the RSRM test site, but those are beyond the scope of the current analysis.

\section{Results}

In this section, Ares I lift-off predictions from Eldred's original, unmodified version of the DSM-2 are compared with predictions using the modifications discussed in Section 3. The only feature of the methodology not discussed in this document is a vehicle "lift-off" simulation. Basically, for both the unmodified and modified methods, the calculations are performed for multiple vehicle elevations in order to simulate lift-off. The sound sources are automatically moved along the exhaust axis as the vehicle position is elevated. Once the vehicle has reached an elevation that all sound sources occur before deflection, a maximum envelope is taken for the entire liftoff event.

Inputs for the DSM-2 model are based on the Ares I first stage ballistics configuration documented by Kibbey. ${ }^{12}$ Some general performance parameters are also shown in Table 1 . Sound pressure level predictions were generated at positions of $4.5 \mathrm{~m}$ and $76 \mathrm{~m}$ from the nozzle exit plane of the Ares I vehicle. 
Table 1. Geometric and Performance Parameters for Ares I. ${ }^{12}$

\begin{tabular}{|r|c|c|}
\hline Number of Nozzles : & \multicolumn{2}{|c|}{1} \\
\hline Exit Diameter : & $12.73 \mathrm{ft}$ & $3.88 \mathrm{~m}$ \\
\hline Exit Velocity : & $8050 \mathrm{ft} / \mathrm{s}$ & $2454 \mathrm{~m} / \mathrm{s}$ \\
\hline Thrust at Sea Level : & $3,310,000 \mathrm{lb}_{\mathrm{f}}$ & $14,700,000 \mathrm{~N}$ \\
\hline
\end{tabular}

The modified DSM-2 predictions are compared with Eldred's original model in Figs. 12 and 13. The modifications described in Section 3 have a significant effect on the DSM-2 results, mainly due to the shorter core length and the "terminated core" procedure at lift-off. When the maximum power source location is moved closer to the vehicle, it causes the sound pressure levels on the vehicle to increase and to shift towards higher frequencies. The RSRM directivity produces different slopes on both side of the peak sound pressure level. This is more noticeable for the vehicle position close to the nozzle exit, as shown in Fig. 12. The drop-off of sound pressure levels is steeper on both sides of the peak level. There is also a noticeable change in slope that occurs roughly at $2000 \mathrm{~Hz}$, which is contributed to the new RSRM directivity indices.

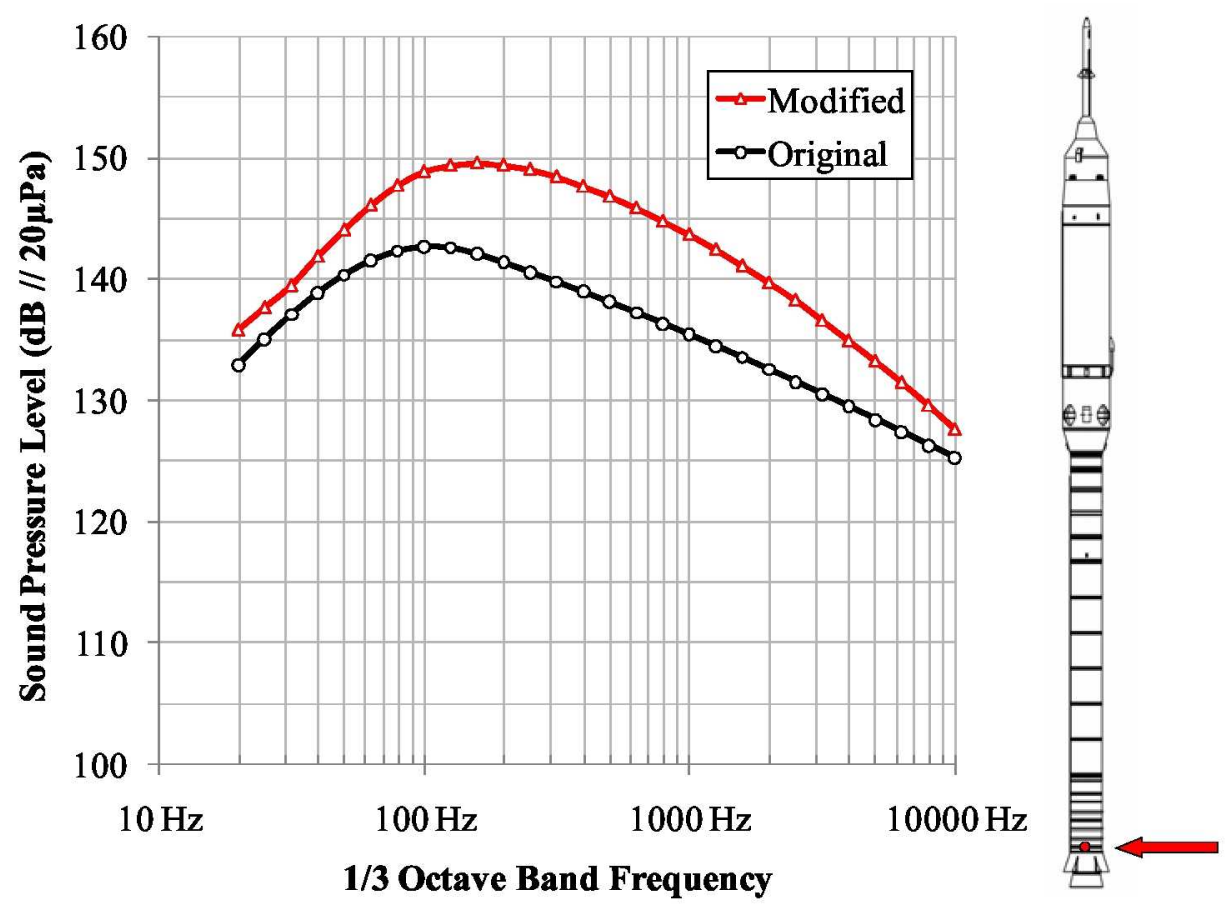

Figure 12. Comparison of predicted SPLs for Ares I, $4.5 \mathrm{~m}$ from the nozzle exit plane. 

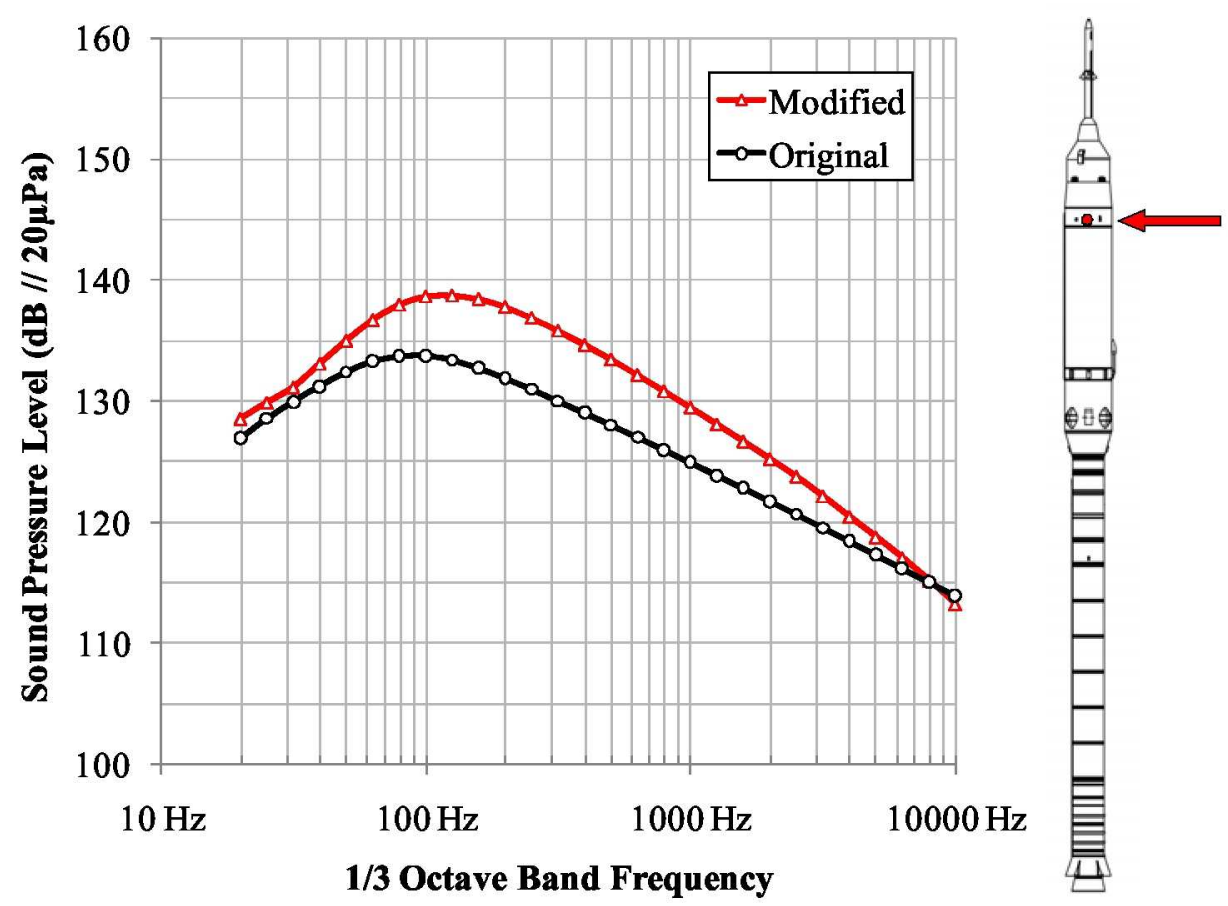

Figure 13. Comparison of predicted SPLs for Ares I, $76 \mathrm{~m}$ from the nozzle exit plane.

\section{Summary and Future Work}

Three modifications were made to the NASA SP-8072 DSM-2 in order to improve its ability to generate useful lift-off acoustic predictions, with special attention focused on Ares I predictions. In the past, many users have found that DSM-2 predictions are 5-10 dB lower than the DSM-1 predictions as well as measured data. This section summarizes the efforts made investigating and correcting some of the discrepancies with this methodology.

In Section IIIA, the potential core length, which is used for sound power distribution, was shortened by a factor of 2. This modification is not unique to this analysis. It has been documented by Varnier, ${ }^{5,6}$ and used by several analysts since that time. For the Ares I analysis, a RANS CFD turbulence model was used to check and compare the sound power distributions of Varnier and Eldred. Observation of the CFD laminar core region provided conclusive evidence that Varnier's core length approximation is more appropriate. This effects the acoustic environment predictions from both undeflected and deflected exhaust flows by shifting the noise sources closer to the nozzle exit, thereby increasing the sound pressure levels on the launch vehicle.

In Section IIIB, a core termination procedure was implemented in for exhaust plumes experiencing impact with a deflector. The most significant discrepancy between Eldred's DSM-1 and DSM-2 methodologies concerned the treatment of source power distribution after deflection. The DSM-1 provided empirical data for discretefrequency source placement for both deflected and undeflected flow; however, the DSM-2 provided no methodology to adjust source location after a flow experiences deflection. If the physical impact of a deflector was ignored, the plume simply experienced a change in direction. This allowed the plume and core structure to be preserved, and forced the peak sound power location too far downstream to attain reliable acoustic environments on the launch vehicle. In order to improve this feature, a RANS CFD turbulence model was used to observe the laminar region of an RSRM plume before and after deflection. The results showed that the potential core was effectively disrupted upon impact with the deflector, and that the laminar-to-turbulent transition occurred immediately downstream of deflection. A new procedure was introduced into the DSM-2 to terminate the potential core at the deflector location, which shifted noise sources much closer to the vehicle. The new procedure was validated using acoustic measurements from multiple Saturn V launches. The results showed clear evidence that the core termination approach was an important improvement to Eldred's original DSM-2 methodology.

In Section IIIC, measured RSRM Directivity Indices were added to Eldred's empirical database specifically for Ares I predictions. The directional trends in the RSRM data compared well with Eldred's data in general; however, the SPLs for frequencies above $500 \mathrm{~Hz}$ showed more directivity than expected. Without further analysis 
and testing, it remains unclear whether the high frequency propagation behavior was caused specifically by the RSRM plume characteristics or by undesired effects of the surrounding terrain.

In Section IV, Ares I lift-off acoustic predictions were presented in order to compare the modified DSM-2 with Eldred's original version. The overall SPL predictions increased by more than $5 \mathrm{~dB}$, and the peak SPL shifted roughly 2 third-octave bands higher in frequency. The "terminated core" modification had less effect on Ares I than on Saturn V predictions because of the difference in original core length between the two. The RSRM Directivity Indices increased the slopes of SPL drop-off on both sides of the peak level.

Future work plans on this subject include: 1) parametric studies using CFD to investigate the trends of potential core and supersonic core lengths with changing exhaust flow properties, 2) further validation of DSM-2 core length modifications using launch acoustic measurements, and 3) further analysis of measured RSRM data for use as empirical references in both the DSM-1 and DSM-2.

\section{Acknowledgments}

The authors are grateful to Jeff West, CFD Team Lead in the Fluid Dynamics Branch at Marshall Space Flight Center, for providing the CFD solutions shown in this paper.

\section{References}

${ }^{1}$ Eldred, K., "Acoustic Loads Generated by the Propulsion System," NASA SP-8072, June 1971.

${ }^{2}$ Lighthill, M. J., "On Sound Generated Aerodynamically, Part I, General Theory," Proceedings of the Royal Society of London, Vol. 211, March 1952.

${ }^{3}$ Lighthill, M. J., "On Sound Generated Aerodynamically, Part II, Turbulence as a Source of Sound," Proceedings of the Royal Society of London, Vol. 222, February 1954.

${ }_{4}^{4}$ Anderson, A. R., Characteristics of Free Supersonic Jets Exhausting into Quiescent Air, Jet Propulsion, Vol. 25, January 1955.

${ }^{5}$ Varnier, J., "Noise Radiated from Free and Impinging Hot Supersonic Jets," AIAA 98-2206, 1998.

${ }^{6}$ Varnier, J., "Experimental Study and Simulation of Rocket Engine Free Jet Noise," AIAA 2001-2207, 2001.

${ }^{7}$ Ogg, G. and White, M., "Prediction of Launch Vehicle Ignition Overpressure and Liftoff Acoustic Environments," Applied Research Associates, ARA Project 5008, Contract NAS8-99079, October 2001.

${ }^{8}$ Tam, C. K. W., "The Sources of Jet Noise: Experimental Evidence," AIAA 2007-3641, 2007.

${ }^{9}$ Haynes, J. M., "Rocket Plume Core Length Studies for Acoustic Predictions," ESTSG-FY08-01266, July 2008.

${ }^{10}$ Krausse, S. C., "Saturn V Aerothermodynamics Flight Evaluation Summary - AS-501 Through AS-503," D5-15796-1, June 1969.

${ }^{11}$ Krausse, S. C., "Saturn V Launch Vehicle Flight Evaluation Report - AS-505, Apollo 10 Mission," MPR-SAT-FE-69-7.

${ }^{12}$ Kibbey, T., "RSRMV-16606 Predicted Performance Report," ATK TR017700, July 2006. 


\section{Modifications to the NASA SP-8072} Distributed Source Method II for Ares I Lift-off Environment Predictions

2009 AIAA Aeroacoustics Conference

Jared M. Haynes

Jacobs ESTS Group

R. Jeremy Kenny

Marshall Space Flight Center 


\section{Outline}

- Introduction

- General Methodology

- Modifications

- Results

- Summary and Future Work 


\section{Introduction}

- NASA currently designing Ares I as a Crew Launch Vehicle.

- Engineers in MSFC Fluid Dynamics Branch responsible for assessing lift-off acoustic levels.

- DSM-2 model in the NASA SP8072 (Eldred, 1971) has been found to have inconsistencies and errors.

- Modifications are made to improve DSM-2 physics and to

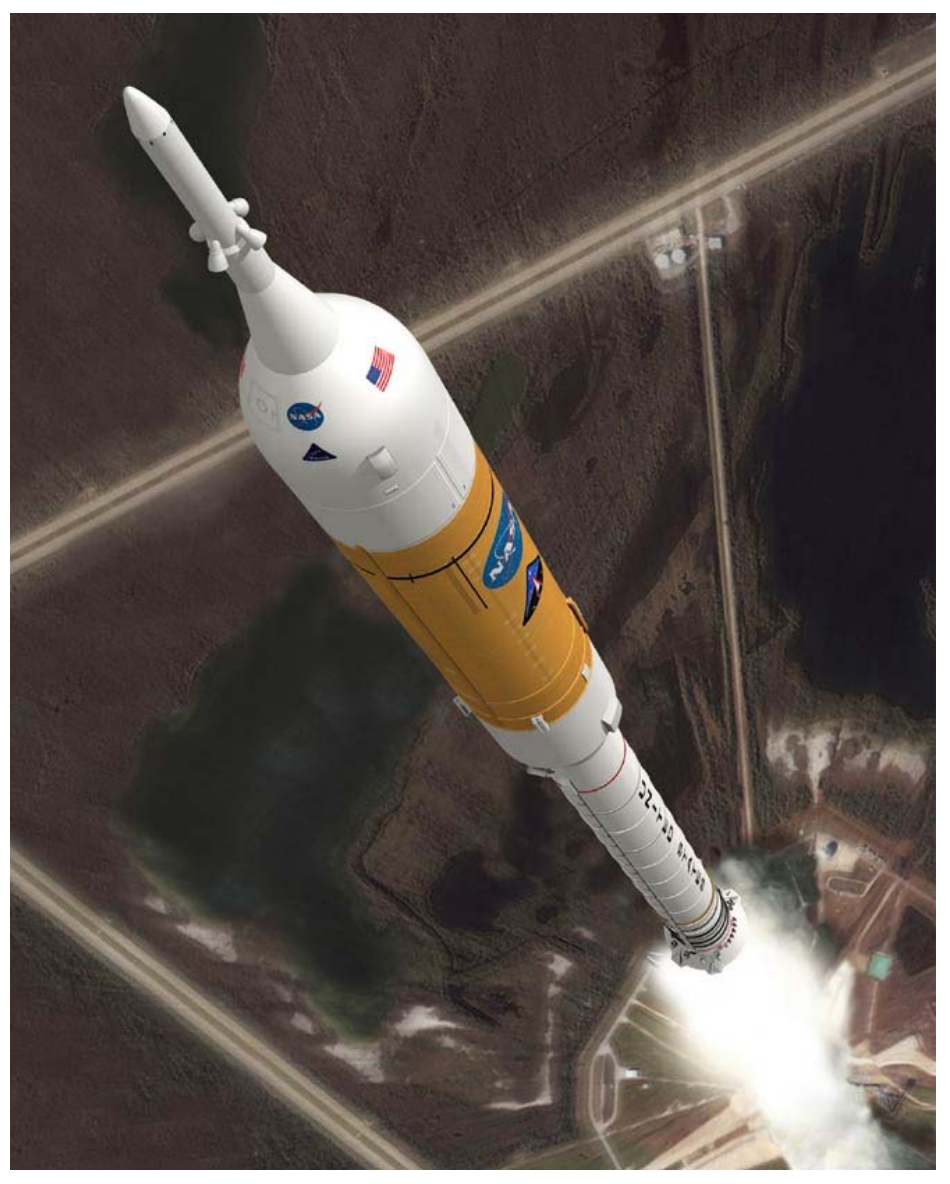
add empirical data specific to Ares I. 


\section{General Methodology}

- Exhaust plume axis defined by geometric configuration at launch.

- Exhaust plume is divided into individual slices, each with length $\Delta \mathrm{x}$.

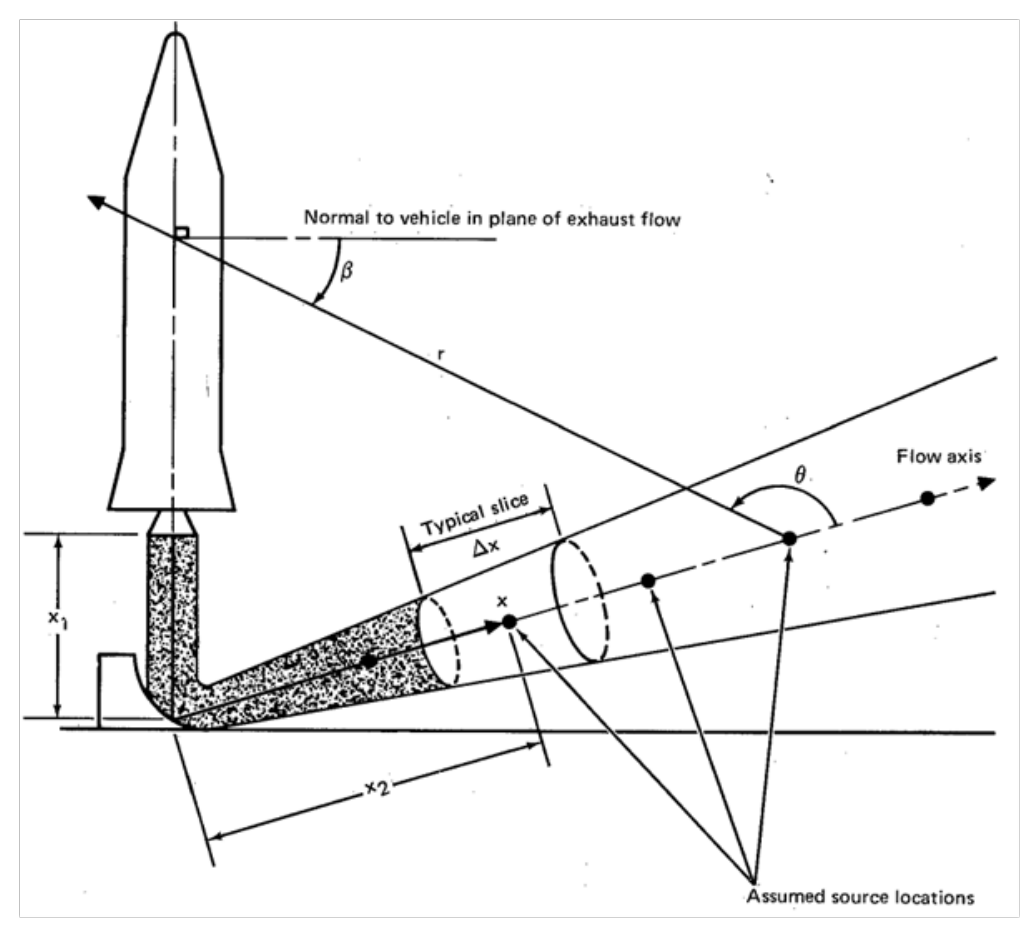

- Overall sound power calculated with kinetic power and acoustic efficiency.

$$
W_{O A} \text { (watts) }=\eta\left(\frac{1}{2} \dot{m} U_{e}^{2}\right) \quad L_{W}(\mathrm{~dB})=10 \log \frac{W_{O A}}{10^{-12} \text { watts }}
$$




\section{General Methodology}

- Sound power for each slice calculated from empirical Non-dimensional Relative Source Power (NRSP) curve.

- Aerodynamic reference is the length of the plume's potential core, $x_{t}$.

- Peak sound power occurs at $1.5 \mathrm{x}_{\mathrm{t}}$.

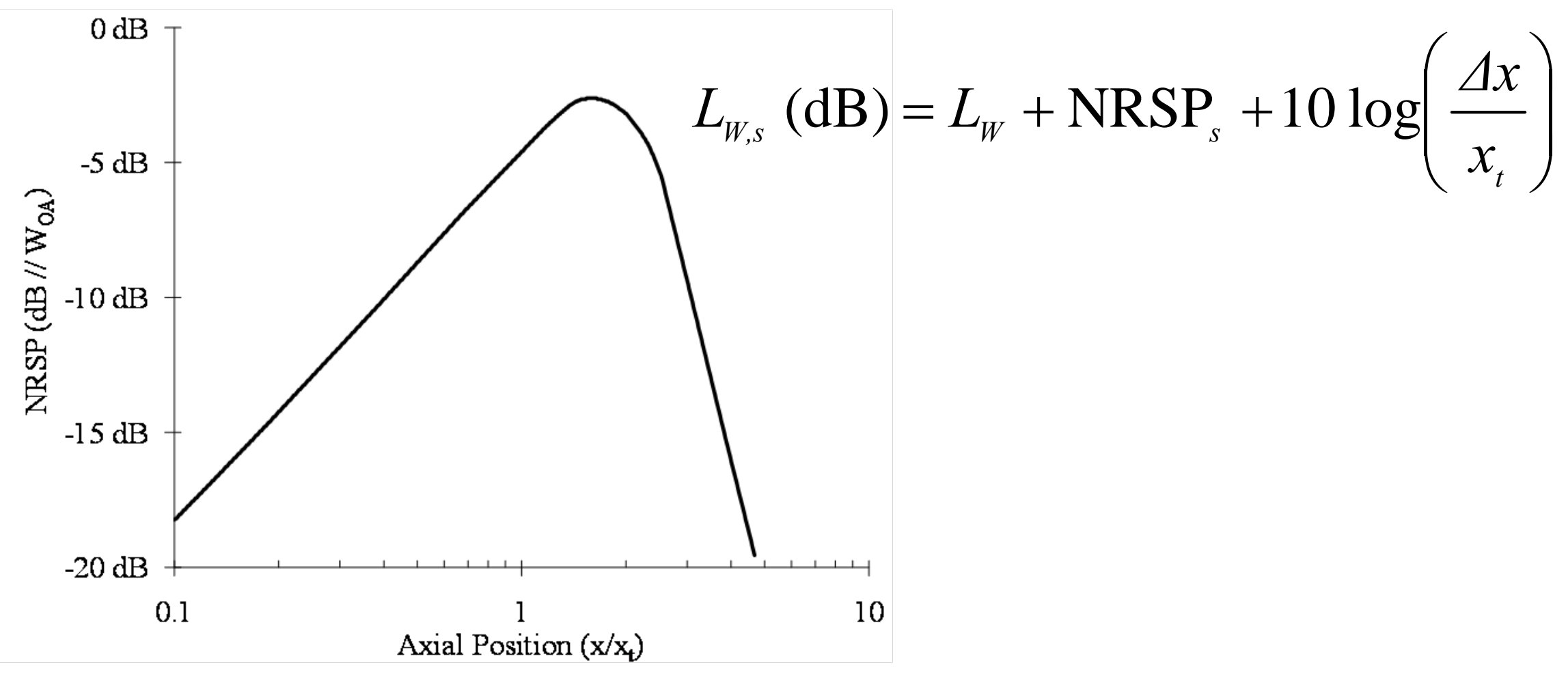




\section{General Methodology}

- Potential core length estimated by curve fit through empirical data.

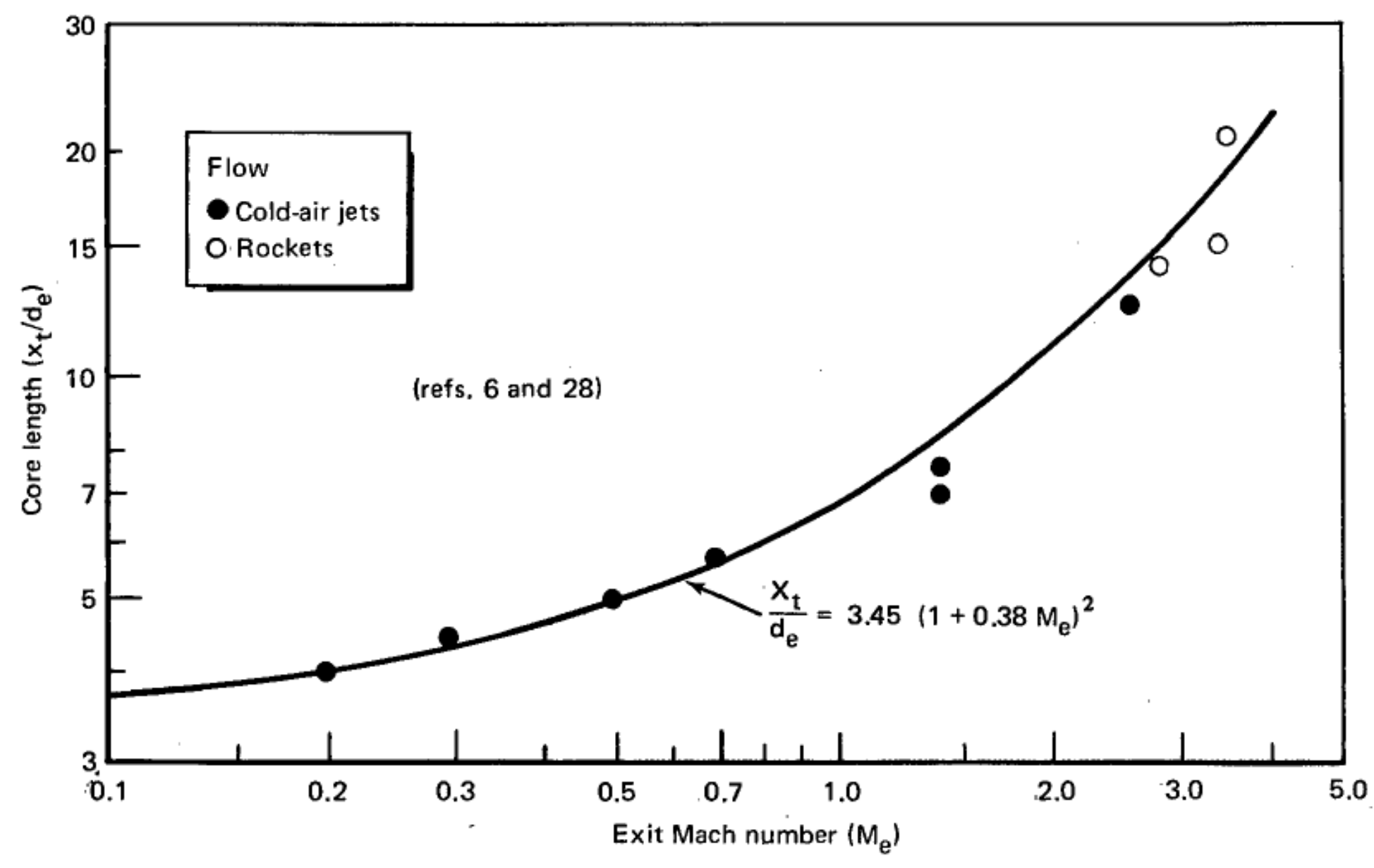




\section{General Methodology}

- Each slice given power spectral content from empirical Nondimensional Relative Sound Power Spectrum (NRSPS) curve.

$$
L_{W, s, b}=L_{W, s}+\operatorname{NRSPS}_{s, b}+10 \log \left(\frac{x a_{e}}{U_{e} a_{o}}\right)+10 \log \Delta f_{b}
$$

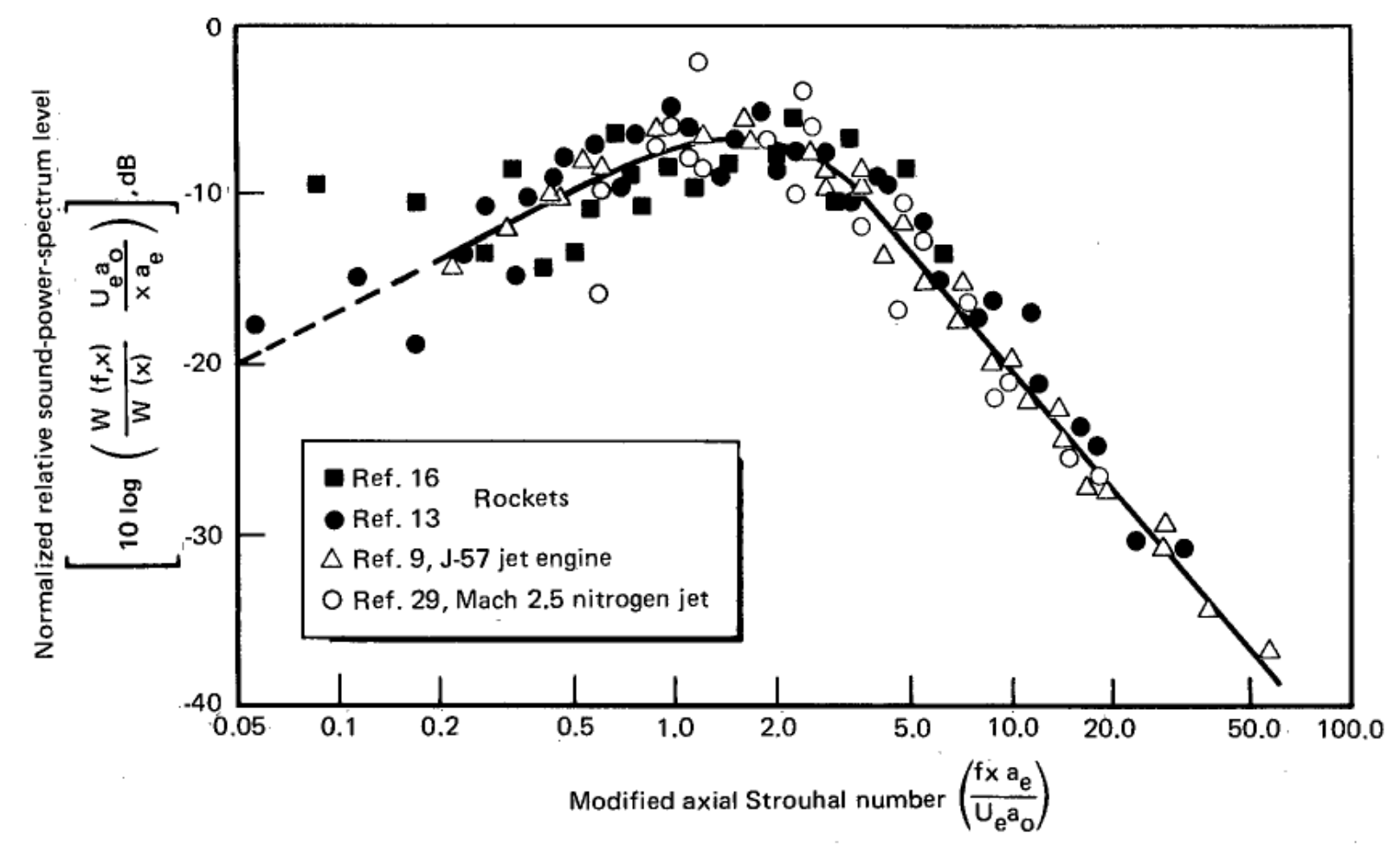




\section{General Methodology}

- Sound pressure level calculated from power contributions of all slices.

- SPL also a function of source distance and Directivity Index.

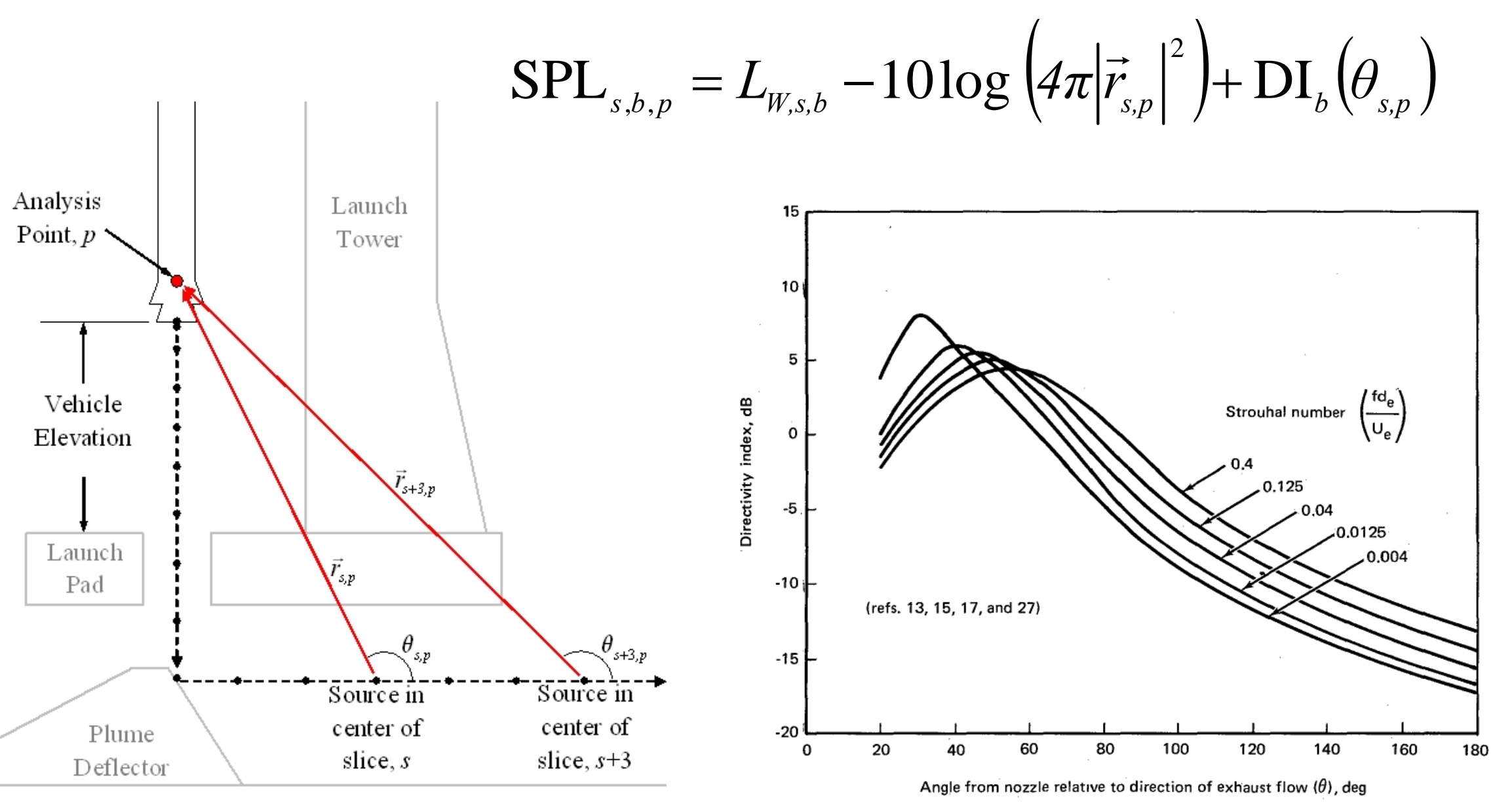




\section{Modification 1: Potential Core}

- Potential core, $x_{t}$, is region of laminar exhaust flow.

- Eldred's approximation is too large for comparable results $\left(2<M_{e}<3\right)$ with horizontal rocket test data (Varnier, 1998).

$$
\frac{x_{t}}{d_{e}}=3.45\left(1+0.38 M_{e}\right)^{2} \quad \text { Eldred }
$$

- Varnier suggested reducing length by a factor of $\sim 2$.

$$
\frac{x_{t}}{d_{e}}=1.75\left(1+0.38 M_{e}\right)^{2} \quad \text { Varnier }
$$

- Better comparisons to horizontal rocket test data with Varnier core length (0-3 $d B$ error) than Eldred (5-7 dB error) (Haynes, 2006-2009 unpublished).

- Possible reason for discrepancy may be in the physics of exhaust plumes with shock patterns, where increased turbulence shortens the length of the laminar region (Shirie, 1967). 


\section{Modification 1: Potential Core}

- RANS CFD code at MSFC used to simulate RSRM exhaust.

- Contour of turbulence kinetic energy used to observe laminar region.

- Sound power distribution from Varnier's core length matches CFD.

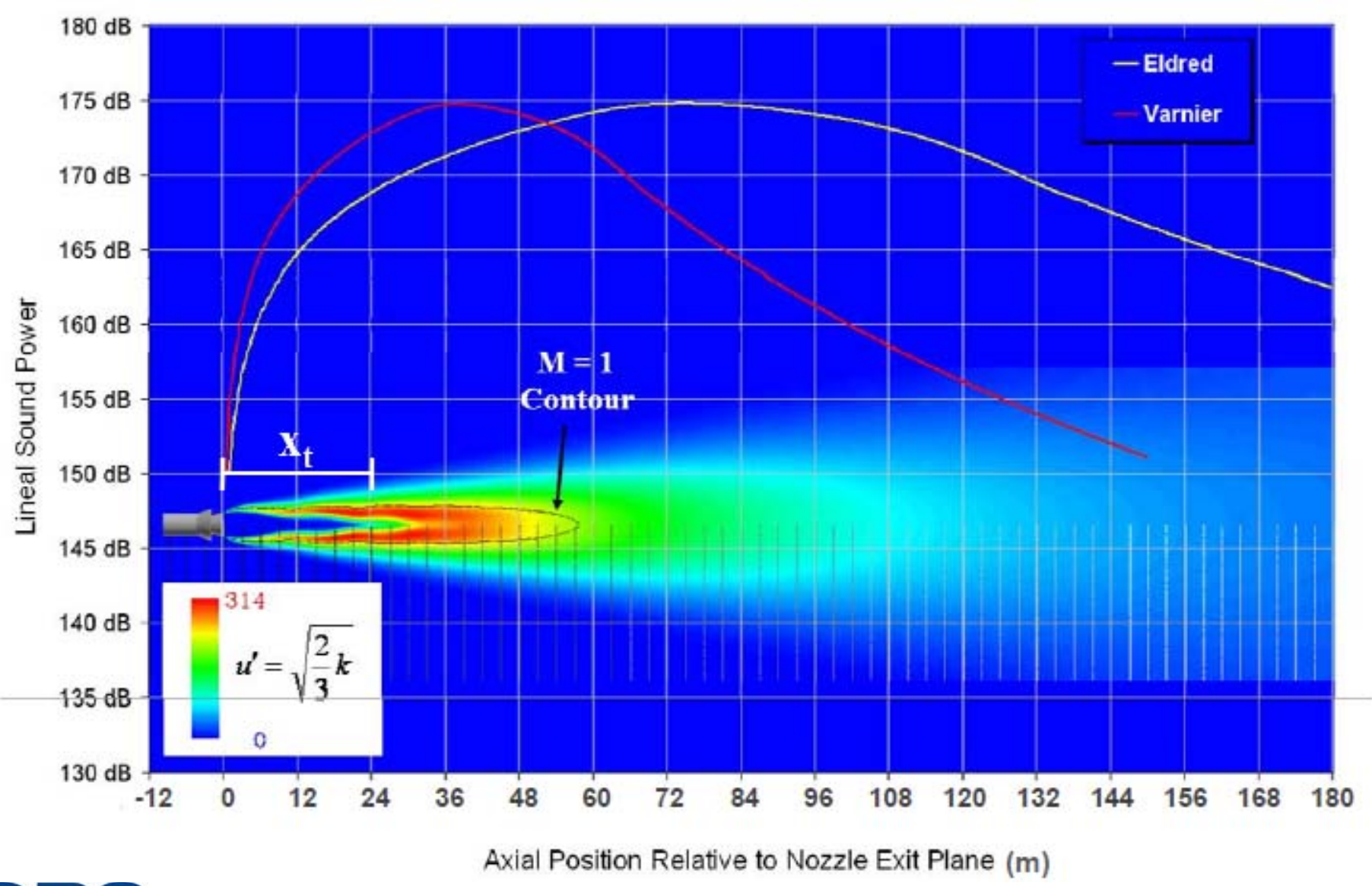




\section{Modification 2: Terminated Core}

DSM-1 Source Locations

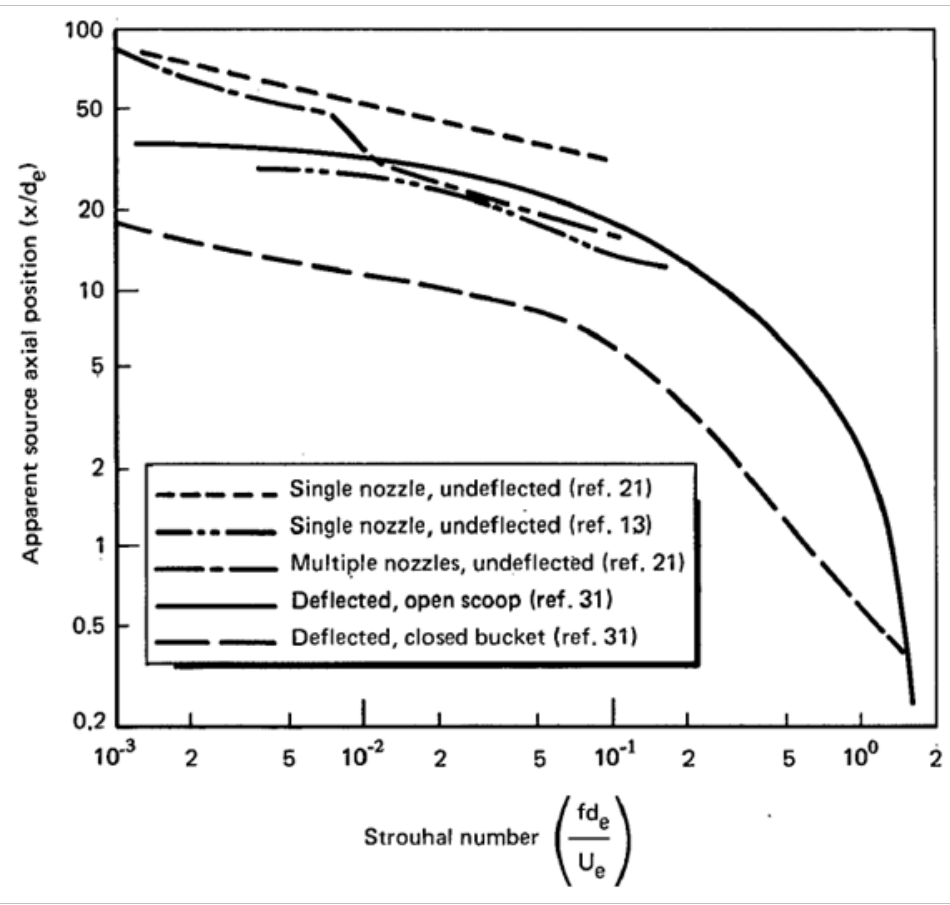

- In DSM-1, Eldred provides empirical source location curves for flows with deflection.

- In DSM-2, no methodology is provided to affect noise source locations with a deflector.

- As written, the DSM-2 simply redirects the plume axis at deflection, preserving the plume structure and axial distance of sources.

- Causes a large discrepancy between DSM-1 and DSM-2 predictions.

- DSM-2 predictions often 5-15 dB lower than measured launch data depending on original undeflected core length. 


\section{Modification 2: Terminated Core}

- RANS CFD code at MSFC used to simulate RSRM exhaust at launch.

- Contour of turbulence kinetic energy used to observe laminar region.

- Laminar core is terminated at the deflector.



AIAA-2009-3160

Page 12 


\section{Modification 2: Terminated Core}

- Terminated core approach implemented into DSM-2.

- Validated with Saturn V launch data.
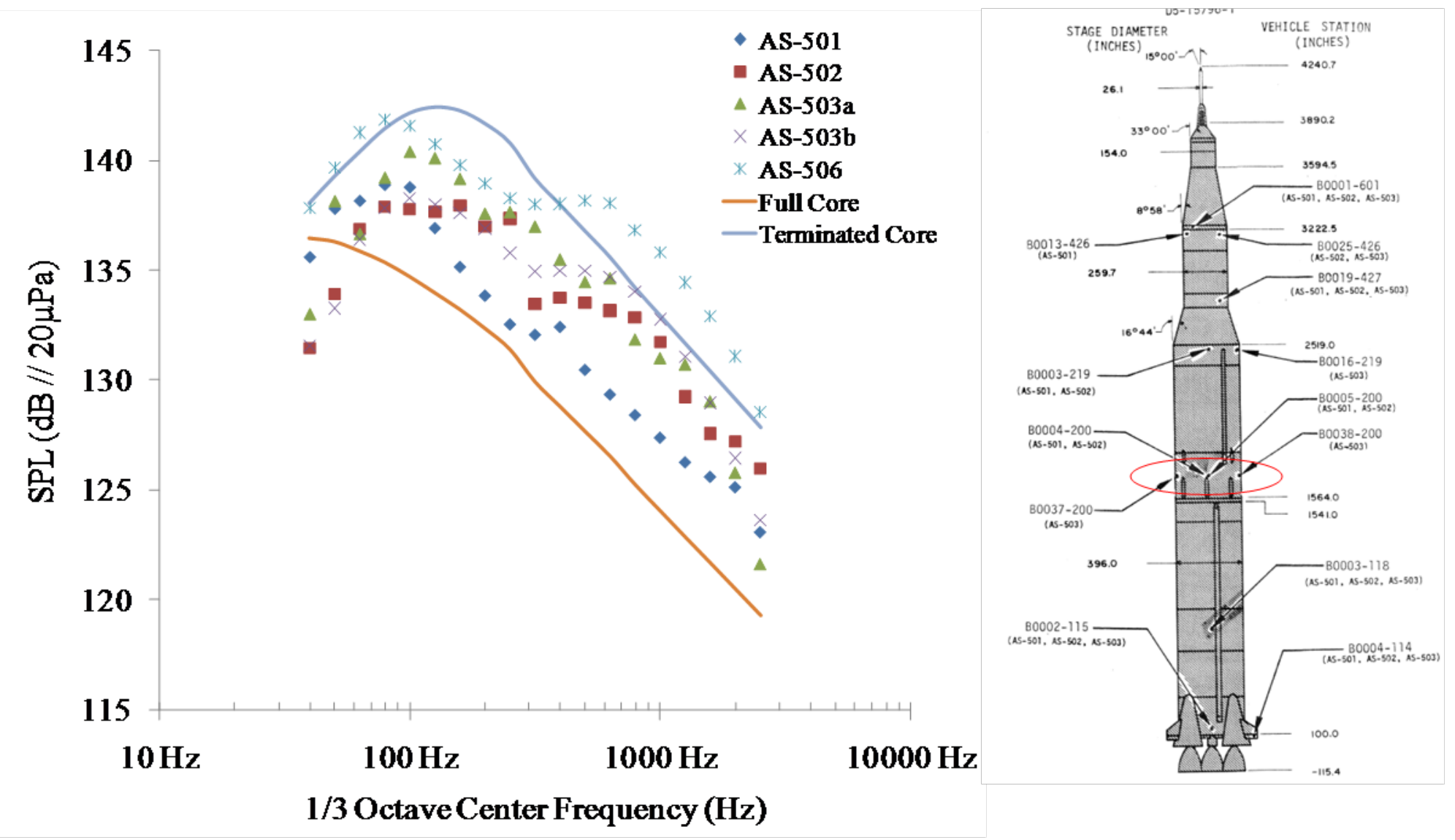


\section{Modification 3: RSRM Directivity}

- Recently collected far-field microphone measurements during 3 RSRM horizontal static tests.

- Used data from only 2 tests for consistency in nozzle gimbaling positions.

- Corrected angular positions by $4^{\circ}$ to account for nozzle vector angle.

- Data used for calculating overall sound power, acoustic efficiency, and directivity indices.

- Implemented RSRM Directivity Index into the DSM-2 model for Ares I predictions.

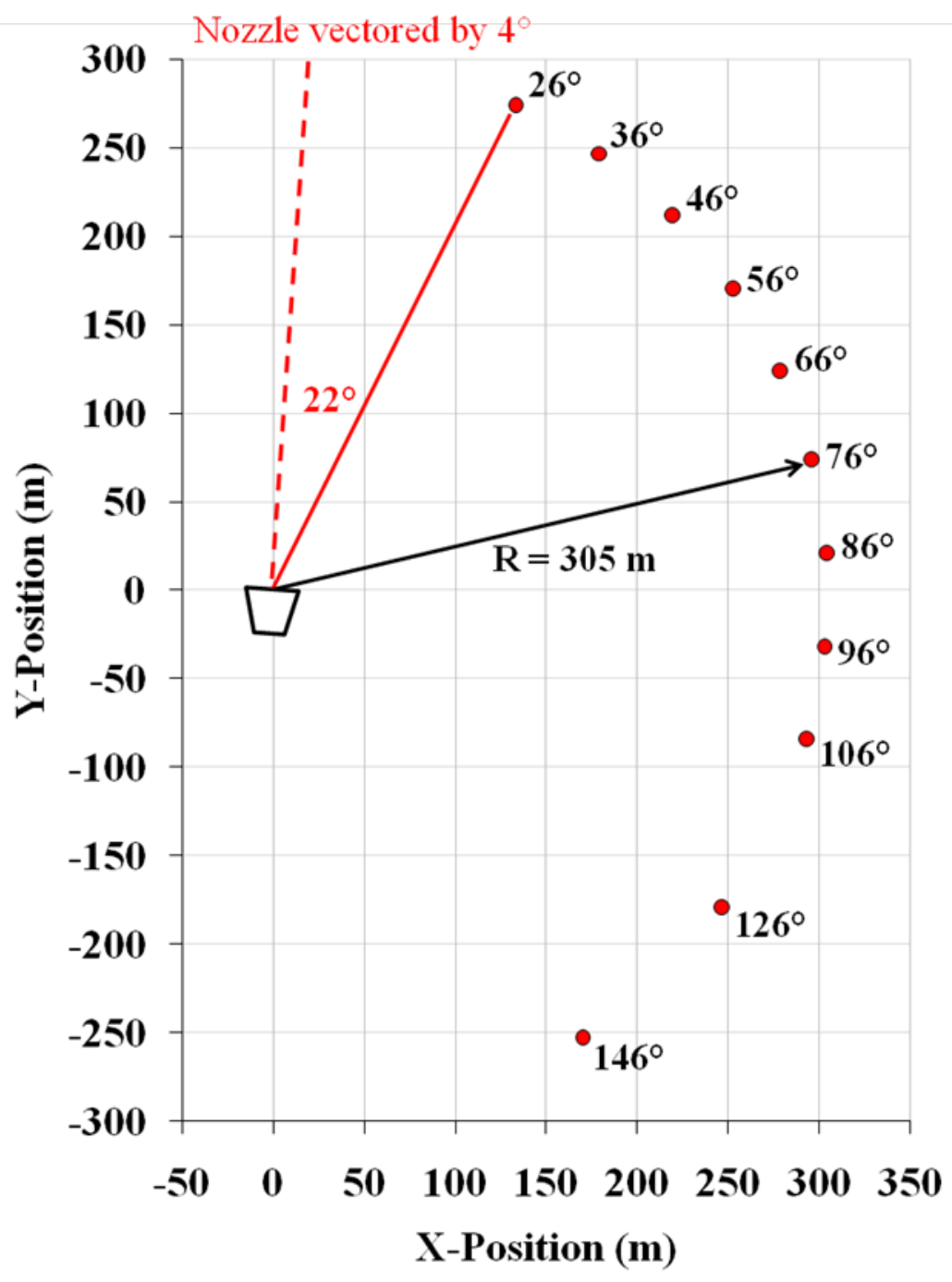




\section{Modification 3: RSRM Directivity}

$$
\mathrm{DI}_{b, \theta}=\mathrm{SPL}_{b, \theta}-10 \log \left(\frac{1}{n} \sum_{i=1}^{n} 10^{\mathrm{SPL}_{b, \theta_{i}} / 10}\right)
$$

Sound Pressure Levels

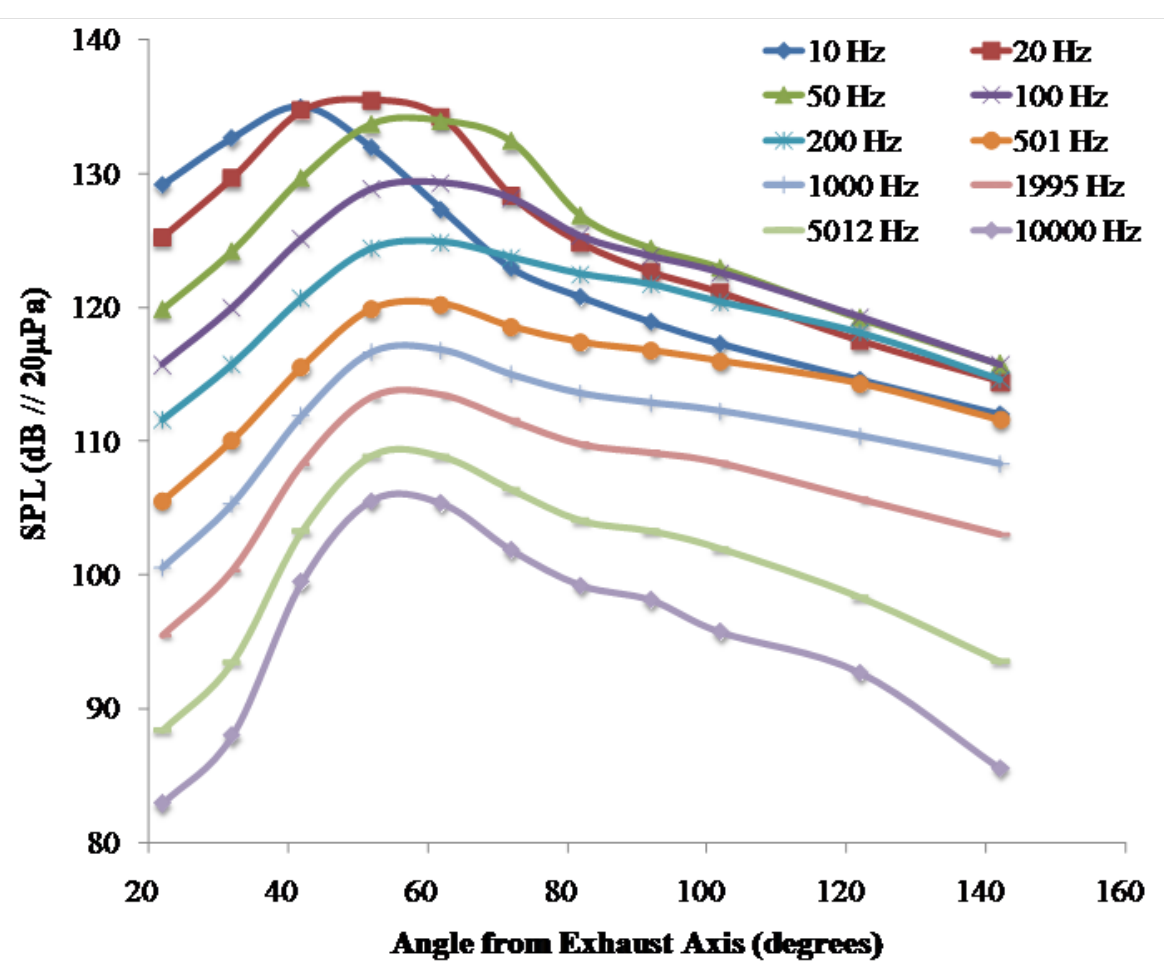

Directivity Indices






\section{Results}

- Predictions compared at $4.5 \mathrm{~m}$ from nozzle exit plane (aft skirt region).

- Overall SPL increased by $7 \mathrm{~dB}$.

- Peak SPL shifted 2 third-octave bands higher in frequency.

- Change in slope of SPL curve from RSRM Directivity Index.

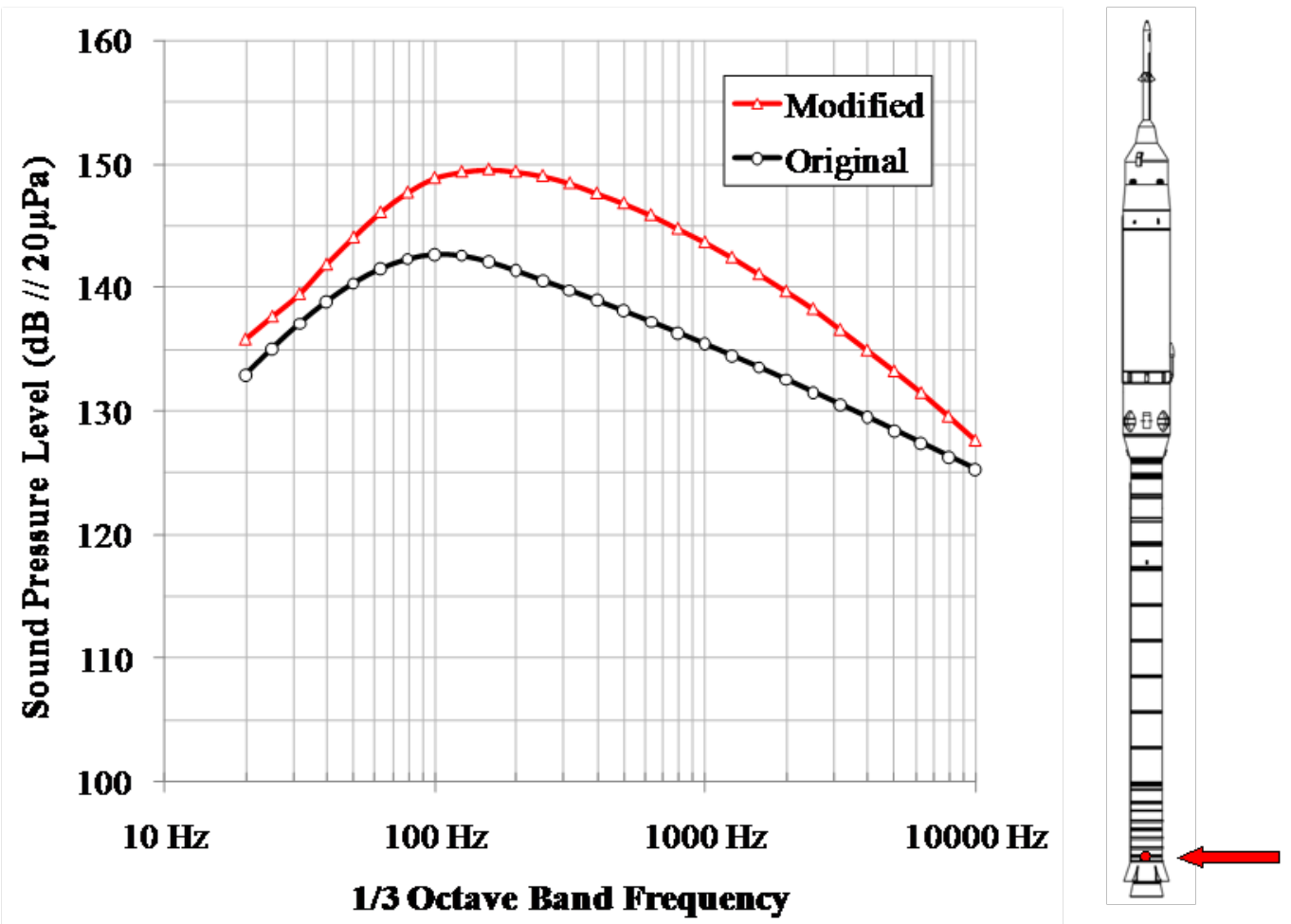




\section{Results}

- Predictions compared at $76 \mathrm{~m}$ from nozzle exit plane (instrument ring).

- Overall SPL increased by $5 \mathrm{~dB}$.

- Peak SPL shifted 2 third-octave bands higher in frequency.

- Change in slope of SPL curve from RSRM Directivity Index.

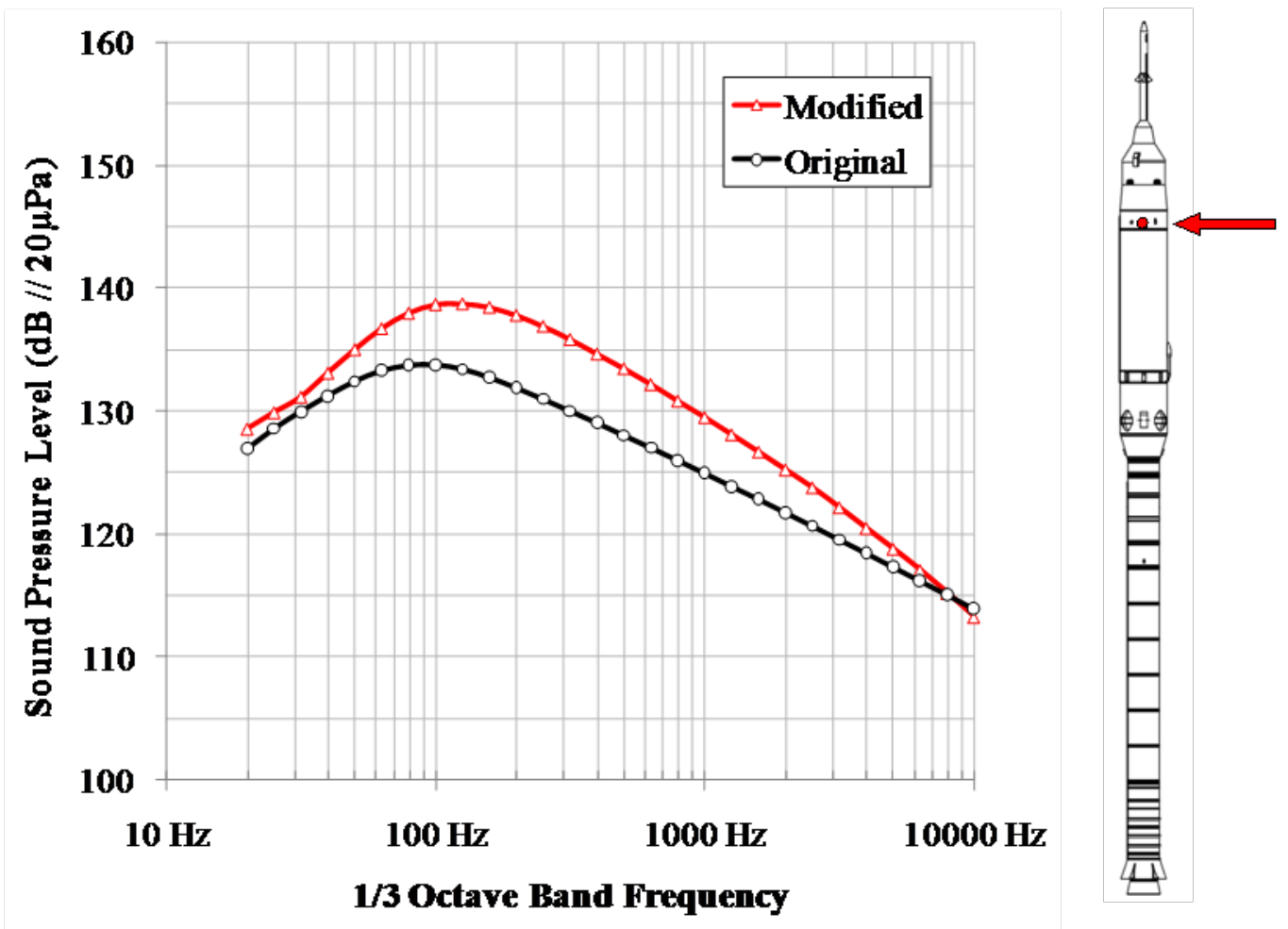




\section{Summary and Future Work}

- Ares I lift-off acoustics predicted using the NASA SP-8072 DSM-2.

- 3 modifications made to the DSM-2.

- Potential core length shortened by a factor of 2 .

- Potential core terminated upon impact with a deflector.

- RSRM acoustic data added to the empirical database.

- CFD and Saturn V lift-off measurements used to validate core length modifications.

- Modified Ares I predictions increased by more than $5 \mathrm{~dB}$ in overall SPL.

- SPL peak shifts to higher frequency by $\sim 2$ third-octave bands.

- Future work includes:

- Use of CFD for parametric studies of the relationship between potential core length and supersonic length with changing nozzle exit conditions.

- Further analysis of RSRM acoustic data. 


\section{Thank You.}

